\title{
A Penalization and Regularization Technique in Shape Optimization Problems in Arbitrary Dimension
}

\author{
Peter Philip* and Dan Tiba ${ }^{\dagger} \ddagger$
}

\begin{abstract}
We consider shape optimization problems, where the state is governed by elliptic partial differential equations (PDE). Using a regularization technique, unknown shapes are encoded via shape functions, turning the shape optimization into optimal control problems for the unknown functions. The method is studied for elliptic PDE to be solved in an unknown region (to be optimized), where the regularization technique together with a penalty method extends the PDE to a larger fixed domain. Additionally, the method is studied for the optimal layout problem, where the unknown regions determine the coefficients of the state equation. In both cases and in arbitrary dimension, the existence of optimal shapes is established for the regularized and the original problem, with convergence of optimal shapes if the regularization parameter tends to zero. Error estimates are proved for the layout problem. In the context of finite element approximations, convergence and differentiability properties are shown. A series of numerical experiments demonstrate the method computationally for an industrially relevant elliptic PDE with two unknown shapes, one giving the region where the PDE is solved, and the other determining the PDE's coefficients.
\end{abstract}

*Department of Mathematics, Ludwig-Maximilians University (LMU) Munich, Theresienstrasse 39, 80333 Munich, Germany, philip@math.lmu.de

$\dagger$ Institute of Mathematics (Romanian Academy), P.O.Box 1-764, RO-014700 Bucharest and Academy of Romanian Scientists, Splaiul Independentei 54, 050094 Bucharest, dan.tiba@imar.ro .

${ }_{\ddagger}^{\ddagger}$ Basque Center for Applied Mathematics, Alameda Mazarredo 14, E-48009 Bilbao, Basque Country, Spain 
Keywords. shape optimization, optimal control, fixed domain method, elliptic partial differential equation, optimal layout problem, error estimate, numerical simulation

\section{Introduction}

A typical shape optimization problem $(P)$ for elliptic equations has the form:

$$
\begin{gathered}
\min _{\Omega} \int_{\Lambda} j\left(x, y_{\Omega}(x)\right) \mathrm{d} x, \\
-\Delta y_{\Omega}=f \quad \text { in } \Omega \\
y_{\Omega}=0 \quad \text { on } \partial \Omega .
\end{gathered}
$$

Here $\Omega$ is some (unknown) bounded open set, $\Omega \subseteq D \subseteq \mathbb{R}^{d}$ or $E \subseteq \Omega \subseteq D \subseteq$ $\mathbb{R}^{d}$, where the given sets $E \subset D$ are also bounded and open, $D$ connected. Moreover, $\Omega \in \mathcal{O}$, where $\mathcal{O}$ denotes the class of all admissible sets (to be specified). In (1.1) - (1.3), $\Lambda$ may be either $\Omega$ or $E$ (in the second case), $f \in L^{2}(D)$, and natural hypotheses are to be imposed on the integrand $j(\cdot, \cdot)$.

Another important type of shape optimization problems associated to elliptic operators is the so-called "optimal layout" problem, looking for the optimal distribution of several materials in the given domain $D \subseteq \mathbb{R}^{d}$, such that the obtained "composition" satisfies certain prescribed properties characterized by a given mapping $y_{d} \in L^{2}(D)$. In this paper, the case of three different materials is discussed, but our method may be easily applied to an arbitrary number of different materials, in arbitrary dimension.

If $\chi_{1}, \chi_{2}, \chi_{3}$ denote the characteristic functions of the regions occupied by each material in $D$, i.e. $\chi_{i}(x) \in\{0,1\}, i=1,2,3, \chi_{1}+\chi_{2}+\chi_{3}=1$, we 
formulate the optimization problem $(R)$ :

$$
\min \int_{D}\left(y-y_{d}\right)^{2} \mathrm{~d} x
$$

(1.5) $\int_{D}\left\{\left(a_{1} \chi_{1}+a_{2} \chi_{2}+a_{3} \chi_{3}\right) \nabla y \cdot \nabla v+a_{0} y v-f v\right\} \mathrm{d} x=0, \forall v \in H^{1}(D)$.

Here each $a_{i}>0$ characterizes the properties of the material " $i$ ", $i=1,2,3$ and $f \in L^{2}(D), a_{0}>0$ are given. On $\partial D$, Neumann boundary conditions are imposed.

In both problems (1.1) - (1.3) and (1.4), (1.5) more general elliptic operators or other boundary conditions may be considered (in the second case), etc. Supplementary constraints may be added on $\Omega, \chi_{i}, i=1,2,3$, the state $y_{\Omega}$, respectively $y$.

The mathematical literature on such problems, using various types of assumptions and methods, is vast: Pironneau [21], Sokolowski and Zolesio [26], Allaire [1], Bendsoe [2], Neittaanmäki, Sprekels and Tiba [16], Sverak [27], Chenais and Zuazua [5], Neittaanmäki and Tiba [17], etc.

A main ingredient of our approach is the use of shape functions defined in $D$ and of a technique for the approximation of characteristic functions previously used in [13], [15]. For instance, if $g$ is continuous in $\bar{D}$, then

$$
\Omega_{g}=\operatorname{int}\{x \in D: g(x) \geq 0\}
$$

is an open Caratheodory set, not necessarily connected. It may even have an infinity of connected components if $g$ has a strongly oscillatory behavior (example in $\left.D=(0,1) \subseteq \mathbb{R}, g(t)=t \sin \frac{1}{t}\right)$.

Notice that $\Omega_{g}$ defined in (1.6) is not necessarily a set of class C (i.e. even the segment property may fail). Some counterexamples may be found in [10], [28]. 
It is possible to assume that $g$ is piecewise continuous in $D$ and, then, $\Omega_{g}$ has similar properties, [20].

Notice as well that (1.6) is essentially different from the "level set method" [18], [23] since no time dependence for the functions $g$ and no "evolution" for the corresponding open sets $\Omega_{g}$, is assumed. No Hamilton-Jacobi equation will be used in our subsequent argument and this allows very weak assumptions on $g, \Omega_{g}$. In fact, we use the term shape functions instead of level functions to underline the difference between the two methods.

If $\Omega_{n} \rightarrow \Omega$ in the complementary Hausdorff-Pompeiu metric, then there are $g_{n}, g \in C(\bar{D})$, such that $\Omega_{n}=\Omega_{g_{n}}, \Omega=\Omega_{g}$ (in the sense of (1.6)) and $g_{n} \rightarrow g$ uniformly in $\bar{D}$, [16]. The converse is false, in general: take $\Omega_{n}=\Omega$, $\forall n \in \mathbb{N}$ and $\Omega=\Omega_{g}$ for some $g \in C(\bar{D}), g \not \equiv 0$; then we may write (for instance) $\Omega_{n}=\Omega_{g_{n}}, g_{n}=\frac{1}{n} g$ and clearly $g_{n} \rightarrow 0$ uniformly in $\bar{D}$ while $g \not \equiv 0$.

In the case of the problem $(1.4),(1.5)$, we shall assume that $g \in H_{\text {loc }}^{1}(D)$, with some additional conditions (see (3.1) - (3.3)). The corresponding level sets $\Omega_{g}=\{x \in D: g(x)>0\}$ are quasi-open sets in the sense of potential theory. Since $D \subseteq \mathbb{R}^{d}$ and $d \in \mathbb{N}$ is arbitrary, they are just measurable subsets.

Let $H: \mathbb{R} \rightarrow \mathbb{R}$ be the Heaviside function. Then $H(g): D \rightarrow \mathbb{R}$ is the characteristic function of $\bar{\Omega}_{g}$ in $D$ (for the second problem, this holds only up to a null set and under the additional assumption that $g$ satisfies (3.1) below with $p_{i}$ replaced by $g$, cf. (3.4)). We denote by $H_{\varepsilon}: \mathbb{R} \rightarrow$ $\mathbb{R}$ a regularization of the Yosida approximation of the maximal monotone extension of $H$ in $\mathbb{R} \times \mathbb{R}$. Then $H_{\varepsilon}(g): D \rightarrow \mathbb{R}$ is an approximation of the characteristic function of $\Omega_{g}$ in $D$, in a certain sense. In the next section, the regularization of the problem (1.1) - (1.3) will be studied, while Section 3 is 
devoted to the problem (1.4), (1.5). In Section 4, error estimates with respect to the penalization/regularization parameter are discussed in both problems. Section 5 is devoted to discretization and further approximation properties. The final section provides an algorithm for numerical implementation, and it contains a series of numerical results, where the algorithm has been applied to an industrially relevant shape optimization problem that combines features of $(1.1)-(1.3)$ and $(1.4),(1.5)$.

\section{Penalization and Regularization}

We fix $\Lambda=E$ in the problem (1.1) - (1.3) and we define its approximation $\left(P_{\varepsilon}\right)(\varepsilon>0)$ by:

$$
\begin{gathered}
\min _{g} \int_{E} j\left(x, y_{\varepsilon}(x)\right) \mathrm{d} x, \\
-\Delta y_{\varepsilon}+\frac{1}{\varepsilon}\left(1-H_{\varepsilon}(g)\right) y_{\varepsilon}=f \quad \text { in } D, \\
y_{\varepsilon}=0 \quad \text { on } \partial D .
\end{gathered}
$$

We choose in this section the regularization $H_{\varepsilon}(\cdot)$ such that $H_{\varepsilon}(r)=1$ for $r \geq 0$.

As mentioned in Section 1, since $\Lambda=E$, we have to impose the constraint

$$
g \geq 0 \quad \text { in } E .
$$

This ensures $E \subseteq \Omega_{g}$ (see (1.6)). It is clear that $y_{\Omega} \in H_{0}^{1}(\Omega)$, the solution of $(1.2),(1.3)$, may always be extended by 0 to $D$ and the cost functional (1.1) makes sense even for $\Omega$ not containing $E$ (respectively without imposing (2.4)). Under this convention, one may always take $\Lambda=D$ instead of $\Lambda=\Omega$. 
However, this procedure cannot be applied for Neumann boundary conditions as in (1.4), (1.5) and we shall not use it.

If $\Lambda=\Omega_{g}$, then the cost functional (2.1) has to be replaced by

$$
\int_{D} H_{\varepsilon}(g(x)) j\left(x, y_{\varepsilon}(x)\right) \mathrm{d} x
$$

and (2.4) is no more necessary, but might still be imposed additionally.

The idea of the approximation $(2.1)-(2.4)$ or $(2.5)$ is that the unknown geometry $\Omega=\Omega_{g}$ is "hidden" in the parametrization $g \in C(\bar{D})$ and the shape optimization problem (1.1) - (1.3) is replaced with a control by the coefficients problem, defined in the given fixed domain $D \subseteq \mathbb{R}^{d}$. Denote by $\emptyset \neq U_{\text {ad }} \subseteq C(\bar{D})$ the family of admissible controls $g$, i.e. satisfying (2.4) and other conditions, if any.

Proposition 2.1. Assume that $\emptyset \neq U_{\mathrm{ad}} \subseteq C(\bar{D})$ is compact and the convergence $j\left(x, y_{n}(x)\right) \rightarrow j(x, y(x))$ weakly in $L^{1}(E)$ if $y_{n} \rightarrow y$ strongly in $H^{2}(D)$. Then the problem $\left(P_{\varepsilon}\right)$ has at least one optimal pair $\left[y_{\varepsilon}^{*}, g_{\varepsilon}^{*}\right] \in$ $\left[H^{2}(D) \cap H_{0}^{1}(D)\right] \times U_{\text {ad }}$ for each $\varepsilon>0$.

Sketch of Proof. For each fixed $\varepsilon>0$, we have the continuity property from the coefficients $g \in U_{\text {ad }}$ to the solutions $y_{\varepsilon}$ of (2.2), (2.3), from the uniform topology in $C(\bar{D})$ to the strong topology of $H^{2}(D) \cap H_{0}^{1}(D)$. Applying this on a minimizing sequence (which exists since $U_{\text {ad }} \neq \emptyset$ ) and using the above assumption on $j(\cdot, \cdot)$, the result is obtained.

Remark. An important example of $U_{\text {ad }}$ satisfying the hypotheses of Prop. 2.1 is given by the signed distance functions that may be associated to any admissible $\Omega \in \mathcal{O}$, where $\mathcal{O}$ is assumed to be compact with respect to the Hausdorff-Pompeiu complementary metric [16, p. 461]. They are uniformly Lipschitzian and they satisfy (2.4) for any $\Omega \supset E$. It is also possible to relax 
the continuity hypotheses on $U_{\text {ad }}$ - this point of view will be stressed in the next section.

Denote $\Omega_{\varepsilon}^{*}:=\Omega_{g_{\varepsilon}^{*}} \in \mathcal{O}$ and let $U_{\text {ad }}$ be given by the signed distance functions associated with the sets in $\mathcal{O}$, where we assume that $\mathcal{O}$ is a compact family of open sets of class C (see [16], [28] for details and examples in this respect).

Theorem 2.2. On a subsequence $\varepsilon \rightarrow 0$, we have $y_{\varepsilon}^{*} \rightarrow y^{*}$ weakly in $H_{0}^{1}(D)$, $\Omega_{\varepsilon}^{*} \rightarrow \Omega^{*} \in \mathcal{O}$ in the complementary Hausdorff-Pompeiu topology, where $\left[\left.y^{*}\right|_{\Omega^{*}}, \Omega^{*}\right]$ is an optimal pair for $(P)$. Here, we assume that $j\left(x, y_{n}(x)\right) \rightarrow$ $j(x, y(x))$ weakly in $L^{1}(E)$ if $y_{n} \rightarrow y$ weakly in $H_{0}^{1}(D)$.

Proof. We have $\Omega_{\varepsilon}^{*} \rightarrow \Omega^{*} \in \mathcal{O}$ on a subsequence, due to the compactness of $\mathcal{O}$. As the shape functions $g_{\varepsilon}^{*}$ are the signed distance functions, we have, on the same subsequence, $g_{\varepsilon}^{*} \rightarrow g^{*}$, where $g^{*}$ is the signed distance function associated to $\Omega^{*},[6]$.

Multiply by $y_{\varepsilon}^{*}$ the equations $(2.2),(2.3)$ associated to $g_{\varepsilon}^{*}$. We get that $\left\{y_{\varepsilon}^{*}\right\}$ is bounded in $H_{0}^{1}(D)$ and we may assume that $y_{\varepsilon}^{*} \rightarrow y^{*}$ weakly in $H_{0}^{1}(D)$ on a subsequence. Moreover, we get

$$
\int_{D}\left(1-H_{\varepsilon}\left(g_{\varepsilon}^{*}\right)\right)\left(y_{\varepsilon}^{*}\right)^{2} \mathrm{~d} x \leq C \varepsilon
$$

with $C>0$ some constant independent of $\varepsilon>0$.

Take $K \subset \subset D \backslash \overline{\Omega^{*}}$ an arbitrary, compactly embedded open subset. We may find $c>0$ such that $\left.g^{*}\right|_{K} \leq-c$. Then $\left.g_{\varepsilon}^{*}\right|_{K} \leq-\frac{c}{2}$ for $\varepsilon$ "small", due to the uniform convergence $g_{\varepsilon}^{*} \rightarrow g^{*}$. It yields $H_{\varepsilon}\left(g_{\varepsilon}^{*}\right)=0$ in $K$, for $\varepsilon \leq \varepsilon_{0}$. By (2.6), we infer

$$
\int_{K}\left(y_{\varepsilon}^{*}\right)^{2} \mathrm{~d} x \leq C \varepsilon
$$


and, consequently, $\left.y^{*}\right|_{K}=0$ a.e. As $K$ is arbitrary in $D-\overline{\Omega^{*}}$, we get $\left.y^{*}\right|_{D-\overline{\Omega^{*}}}=0$ a.e.

As $\Omega^{*}$ is of class $\mathrm{C}$, by the Hedberg-Keldys stability property [16], we obtain that $\left.y^{*}\right|_{\Omega^{*}} \in H_{0}^{1}\left(\Omega^{*}\right)$.

Take $\varphi \in C_{0}^{\infty}\left(\Omega^{*}\right)$ arbitrary. By the $\Gamma$-property [16, p. 465], we have $\varphi \in C_{0}^{\infty}\left(\Omega_{\varepsilon}^{*}\right)$, for $\varepsilon \leq \varepsilon_{\varphi}$. Then $\varphi$ may be used as a test function in (2.2) and we have $H_{\varepsilon}\left(g_{\varepsilon}^{*}(x)\right)=1$ on $\operatorname{supp} \varphi \subseteq \Omega_{\varepsilon}^{*}$ by the property that $H_{\varepsilon}(r)=1$ for $r \geq 0$, specific to the definition of $\left(P_{\varepsilon}\right)$ in this section. Then the penalization term in (2.2), multiplied by $\varphi$, vanishes and we obtain

$$
\int_{D} \nabla y_{\varepsilon}^{*} \cdot \nabla \varphi=\int_{D} f \varphi, \quad \forall \varphi \in C_{0}^{\infty}\left(\Omega^{*}\right), \varepsilon \leq \varepsilon_{\varphi} .
$$

One can pass to the limit in (2.7) to see that $\left.y^{*}\right|_{\Omega^{*}} \in H_{0}^{1}\left(\Omega^{*}\right)$ is the solution of $(1.2),(1.3)$.

We now use the obvious inequality

$$
\int_{E} j\left(x, y_{\varepsilon}^{*}(x)\right) \mathrm{d} x \leq \int_{E} j\left(x, y_{\varepsilon}(x)\right) \mathrm{d} x
$$

for each solution $y_{\varepsilon} \in H_{0}^{1}(D)$ of $(2.2)$, (2.3) associated to an arbitrary $g \in U_{\text {ad }}$. Repeating the above argument, we get that, on a subsequence, $y_{\varepsilon} \rightarrow y_{g}$ weakly in $H_{0}^{1}(D)$, and $\left.y_{g}\right|_{\Omega_{g}} \in H_{0}^{1}\left(\Omega_{g}\right)$ is exactly the solution of (1.2), (1.3) in $\Omega_{g}$. Then the assumption on $j(\cdot, \cdot)$ yields $j\left(x, y_{\varepsilon}(x)\right) \rightarrow j\left(x, y_{g}(x)\right)$ weakly in $L^{1}(E)$. One can pass to the limit in $(2.8)$ to show the optimality of $\left[y^{*}, \Omega^{*}\right]$ as claimed.

Remark. If general shape functions from $C(\bar{D})$ are allowed in the definition of $\left(P_{\varepsilon}\right)$, no convergence properties may be established. For instance, it is even possible that $\left\{g_{\varepsilon}\right\}$ is an unbounded sequence (example: $g_{\varepsilon}=\frac{1}{\varepsilon} g, 0 \not \equiv$ $g \in C(\bar{D})$ given). This is due to the nonuniqueness of the parametrization of $\Omega \in \mathcal{O}$ by shape functions. 
Remark. The above convergence result shows that the optimal control $g_{\varepsilon}^{*}$ found in problem $\left(P_{\varepsilon}\right)$ depends in fact on the original geometric optimization problem $(P)$, which is a natural property in this setting.

\section{The Optimal Layout Problem}

In this section, we develop a similar regularization approach for the problem (1.4), (1.5). We consider again representations of the characteristic functions $\chi_{i}, i=1,2,3$ via the Heaviside mapping: $\chi_{1}=H\left(p_{1}\right), \chi_{2}=H\left(p_{2}\right), \chi_{3}=1-$ $H\left(p_{1}\right)-H\left(p_{2}\right)$. It is enough to work with two shape functions $p_{1}, p_{2} \in H_{\text {loc }}^{1}(D)$ and the condition $\chi_{1}+\chi_{2}+\chi_{3}=1$ is automatically fulfilled.

On the pair of shape functions, the following compactness and compatibility hypotheses are imposed:

$$
\begin{aligned}
& \left|p_{i}(x)\right|+\left|\nabla p_{i}(x)\right|_{\mid \mathbb{R}^{d}} \geq \nu>0 \text { a.e. in } D, i=1,2, \\
& \qquad\left|p_{i}\right|_{H^{1+\theta_{Q}^{i}(Q)}} \leq M_{Q}^{i}, \theta_{Q}^{i}>0, i=1,2 \\
& \text { for each } Q \subseteq D \text { compactly embedded open subset, } \\
& \qquad p_{i}(x) \geq 0 \Rightarrow p_{j}(x) \leq 0 \quad i, j=1,2, i \neq j
\end{aligned}
$$

The corresponding level sets are just measurable as $D \subseteq \mathbb{R}^{d}$ and $d \in \mathbb{N}$ is arbitrary.

An important observation is that, by the classical result of Stampacchia [3, p. 195], (3.1) implies

$$
\mu\left(\left\{x \in D: p_{i}(x)=0\right\}\right)=0, \quad i=1,2,
$$

where $\mu(\cdot)$ is the Lebesgue measure in $\mathbb{R}^{d}$. Then, due to $(3.3) ; H\left(p_{1}\right), H\left(p_{2}\right)$, and $1-H\left(p_{1}\right)-H\left(p_{2}\right)$ are indeed characteristic functions of disjoint regions in $D$. 
One can further regularize $H$ (not necessarily in the same way as in Section 2). Here, we impose the requirement

$$
H_{\varepsilon}(r)=0 \quad \text { for } r \leq 0 \text {. }
$$

We define the approximation via regularization of the problem $(R)$ given by (1.4), (1.5) by the problem $\left(R_{\varepsilon}\right)$ :

$$
\begin{gathered}
\min _{\left[p_{1}, p_{2}\right] \in U_{\text {ad }}} \int_{D}\left|y_{\varepsilon}-y_{d}\right|^{2} \mathrm{~d} x, \\
\int_{D}\left\{\left[a_{1} H_{\varepsilon}\left(p_{1}\right)+a_{2} H_{\varepsilon}\left(p_{2}\right)+a_{3}\left(1-H_{\varepsilon}\left(p_{1}\right)-H_{\varepsilon}\left(p_{2}\right)\right)\right] \nabla y_{\varepsilon} \cdot \nabla v\right. \\
\left.+a_{0} y_{\varepsilon} v-f v\right\} \mathrm{d} x=0, \quad \forall v \in H^{1}(D) .
\end{gathered}
$$

We have denoted by $U_{\text {ad }} \subseteq H_{\text {loc }}^{1}(D)^{2}$ the set of admissible pairs of shape functions defined by (3.1) - (3.3).

Remark. It is clear that the same approximation procedure may be applied to $n$ materials, by using $n-1$ shape functions. The compatibility condition (3.3) has to be written in the form $p_{i} \geq 0 \Rightarrow p_{j} \leq 0, \forall j \neq i$.

We also notice that both $(R)$ and $\left(R_{\varepsilon}\right)$ have a fixed domain formulation, although the unknowns are the regions $\Omega_{i}, i=1,2,3$ corresponding to each material. As in the previous section, the aim is to replace the geometric unknowns by analytic unknowns $\left[p_{1}, p_{2}\right] \in U_{\text {ad }}$.

Theorem 3.1. Under the above assumptions, the problem $\left(R_{\varepsilon}\right)$ has at least one optimal triple $\left[y_{\varepsilon}^{*}, p_{1 \varepsilon}^{*}, p_{2 \varepsilon}^{*}\right] \in H^{1}(D) \times U_{\mathrm{ad}}$. On a subsequence, we have

$$
\begin{aligned}
& y_{\varepsilon}^{*} \rightarrow y^{*} \quad \text { weakly in } H^{1}(D), \\
& p_{1 \varepsilon}^{*} \rightarrow p_{1}^{*} \quad \text { in } H_{\mathrm{loc}}^{1}(D), \\
& p_{2 \varepsilon}^{*} \rightarrow p_{2}^{*} \quad \text { in } H_{\mathrm{loc}}^{1}(D),
\end{aligned}
$$


where $\left[y^{*}, p_{1}^{*}, p_{2}^{*}\right] \in H^{1}(D) \times U_{\mathrm{ad}}$ is an optimal triple for the problem $(R)$ by identifying $p_{1}^{*}$, $p_{2}^{*}$ with the measurable sets $\Omega_{1}^{*}=\left\{x \in D: p_{1}^{*} \geq 0\right\}$, $\Omega_{2}^{*}=\left\{x \in D: p_{2}^{*} \geq 0\right\}$, respectively.

Proof. Due to (3.1) - (3.3), we may take subsequences such that (3.9), (3.10) are fulfilled and $\left[p_{1}^{*}, p_{2}^{*}\right] \in U_{\text {ad }}$. Here (3.4) and the a.e. convergence of $p_{i \varepsilon}^{*}$ and $\nabla p_{i \varepsilon}^{*}$ give the argument.

Fix $v=y_{\varepsilon}^{*}$ in (3.7). The ellipticity gives that $\left\{y_{\varepsilon}^{*}\right\}$ is bounded in $H^{1}(D)$ and we may assume $y_{\varepsilon}^{*} \rightarrow y^{*}$ weakly in $H^{1}(D)$ on a subsequence.

The set $\left\{x \in D: p_{i}^{*}(x)=0\right\}$ has zero measure, $i=1,2$. Then, a.e. $D$, we may assume $p_{i}^{*}(x)>0$ or $p_{i}^{*}(x)<0, i=1,2$. In the first case, by the a.e. convergence, we infer $p_{i \varepsilon}^{*}(x)>c$ for each $\varepsilon<\varepsilon_{i}(x)$, and $H_{\varepsilon}\left(p_{i \varepsilon}^{*}(x)\right)=1$ for each $\varepsilon<\varepsilon_{i}(x), i=1$, 2. Similarly, if $p_{i}^{*}(x)<0$, then, for $\varepsilon<\overline{\varepsilon_{i}}(x)$, we obtain $H_{\varepsilon}\left(p_{i \varepsilon}^{*}(x)\right)=0$. Here, we use (3.5) and the fact that one may suppose $H_{\varepsilon}(r)=1$, for $r>\varepsilon$ (see (4.2) as well).

Consequently, on a subsequence, $H_{\varepsilon}\left(p_{i \varepsilon}^{*}\right) \rightarrow H\left(p_{i}^{*}\right)$ a.e. in $D, i=1,2$ and $H\left(p_{i}^{*}\right)$ are indeed characteristic functions in $D$. By the Lebesgue theorem, the convergence is valid in $L^{q}(D), 1 \leq q<\infty$.

One can pass to the limit in (3.7): The observation is that $\left[a_{1} H_{\varepsilon}\left(p_{1 \varepsilon}^{*}\right)+\right.$ $\left.a_{2} H_{\varepsilon}\left(p_{2 \varepsilon}^{*}\right)+a_{3}\left(1-H_{\varepsilon}\left(p_{1 \varepsilon}^{*}\right)-H_{\varepsilon}\left(p_{2 \varepsilon}^{*}\right)\right)\right] \nabla y_{\varepsilon}^{*}$ is bounded in $L^{2}(D)^{d}$ and it converges weakly in $L^{1}(D)^{d}$ to $\left[a_{1} H\left(p_{1}^{*}\right)+a_{2} H\left(p_{2}^{*}\right)+a_{3}\left(1-H\left(p_{1}^{*}\right)-H\left(p_{2}^{*}\right)\right)\right] \nabla y^{*}$. Then the weak limit in $L^{2}(D)^{d}$ is the same and we get that $\left[y^{*}, p_{1}^{*}, p_{2}^{*}\right]$ satisfy the state system (1.5) for $(R)$.

We also have $\int_{D}\left|y_{\varepsilon}^{*}-y_{d}\right|^{2} \mathrm{~d} x \rightarrow \int_{D}\left|y^{*}-y_{d}\right|^{2} \mathrm{~d} x$ and the same argument as at the end of Theorem 2.2 shows that $\left[y^{*}, p_{1}^{*}, p_{2}^{*}\right]$ is an optimal solution for $(R)$.

Remark. More general cost functionals, for instance integral functionals de- 
fined on smooth manifolds of codimension one, are possible to be studied in this setting.

Remark. One may add supplementary constraints in the definition of $U_{\text {ad }}$. For instance, if a certain subset $\omega \subseteq D$ should be made, for various reasons, of material " $i$ ", then it is enough to impose the condition

$$
\left.p_{i}\right|_{\omega} \geq 0
$$

If the total quantity of material " $i$ " is limited, but its distribution is free, then we may ask

$$
\int_{D} H\left(p_{i}\right) \mathrm{d} x \leq k,
$$

where $k>0$ is given, etc.

\section{Estimates with Respect to $\varepsilon>0$}

For any $g \in H_{\text {loc }}^{1}(D)$, we denote by

$$
D_{\varepsilon}(g):=\left\{x \in D: H_{\varepsilon}(g(x)) \neq H(g(x))\right\}
$$

a measurable subset of $D$, defined up to a subset of measure 0 .

Proposition 4.1. Under hypotheses (3.1) for $g \in H_{\mathrm{loc}}^{1}(D)$, we have:

$$
\mu\left(D_{\varepsilon}(g)\right) \rightarrow 0
$$

for $\varepsilon \rightarrow 0$, where, as in (3.4), $\mu(\cdot)$ is the Lebesgue measure in $\mathbb{R}^{d}$.

Proof. For all the regularizations $H_{\varepsilon}(\cdot)$ considered in the previous sections, we may impose that

$$
D_{\varepsilon}(g) \subseteq E_{\varepsilon}(g):=\{x \in D:|g(x)|<\varepsilon\},
$$


without loss of generality.

We notice that, if $\varepsilon_{1}<\varepsilon_{2}$, then $E_{\varepsilon_{1}}(g) \subseteq E_{\varepsilon_{2}}(g)$.

Consequently, $E_{\varepsilon}(g)$ is a decreasing set sequence for $\varepsilon \rightarrow 0$, and

$$
\begin{gathered}
E(g):=\lim _{\varepsilon \rightarrow 0} E_{\varepsilon}(g)=\bigcap_{\varepsilon>0} E_{\varepsilon}(g), \\
\mu(E(g))=\lim _{\varepsilon \rightarrow 0} \mu\left(E_{\varepsilon}(g)\right) .
\end{gathered}
$$

Then the result follows from (3.4) and (4.2) by contradiction.

Assume now that $D \subseteq \mathbb{R}$, i.e. $d=1$.

Proposition 4.2. Let $K_{\mathrm{ad}}$ be compact in $C^{1}(\bar{D})$ and (3.1) be satisfied by each $p \in K_{\mathrm{ad}}$. Then there exists some natural number $\widehat{n}$ such that

$$
Z(p) \leq \widehat{n}, \quad \forall p \in K_{\mathrm{ad}},
$$

where $Z(p)$ denotes the number of zeros of $p \in K_{\mathrm{ad}}$.

Proof. By contradiction, assume there is $\left\{p_{n}\right\} \subseteq K_{\text {ad }}$ such that $Z\left(p_{n}\right) \rightarrow \infty$ for $n \rightarrow \infty$.

On a subsequence, we have $p_{n} \rightarrow p \in K_{\mathrm{ad}}$, uniformly in $\bar{D}$ (by assumption).

Write the zeros of $p_{n}$ (or some subset of them) in increasing order:

$$
\left\{x_{n}^{1}, x_{n}^{2}, \ldots, x_{n}^{i}, \ldots\right\}, \quad i \leq Z\left(p_{n}\right) .
$$

For any fixed $i$, we consider the sequence $\left\{x_{n}^{i}\right\}$ (which may start from some higher order $n$ ). As $\bar{D}$ is compact, on a subsequence, we have $x_{n}^{i_{n}}-x_{n}^{i_{n-1}} \rightarrow 0$, $n \rightarrow \infty$, for certain roots of $p_{n}$.

In certain intermediary points $\tau_{n}$ between the above roots we also have $p_{n}^{\prime}\left(\tau_{n}\right)=0$. On a subsequence, all these points have the same limit $\bar{x}$.

We clearly get $p(\bar{x})=p^{\prime}(\bar{x})=0$, which contradicts (3.1) and ends the proof. 
Proposition 4.3. Under the above hypotheses, we have

$$
\mu\left(D_{\varepsilon}(g)\right) \leq \mu\left(E_{\varepsilon}(g)\right) \leq C \varepsilon,
$$

with $C>0$ a constant independent of $\nu>\varepsilon>0$ and of $g \in K_{\mathrm{ad}}$.

Proof. $E_{\varepsilon}(g)$ is an open subset of $D$ and consists of a union of disjoint open intervals contained in $D$. The two "extreme" intervals (to the left and to the right) may have the same endpoints as $\bar{D}$.

We notice that $g^{\prime} \neq 0$ in $E_{\varepsilon}(g)$, otherwise (3.1) is contradicted (for $\varepsilon<\nu$ ). Since $g \in C^{1}(\bar{D}), g^{\prime}$ has a constant sign in each connected component of $E_{\varepsilon}(g)$, i.e. $g$ is strictly monotone in each subinterval of $E_{\varepsilon}(g)$ and its slope satisfies $\left|g^{\prime}(x)\right| \geq \nu-\varepsilon, \forall x \in E_{\varepsilon}(g)$. Each such subinterval has a maximal character in the sense that $|g(x)|=\varepsilon$ in its endpoints (with the possible exception of the outermost subintervals).

It yields that each interior subinterval contains one zero of $g$ and only one. Therefore, their number is limited by $\widehat{n}+2$ (the maximal number of roots from Prop. 4.2 plus, possibly, two untypical intervals that may occur toward the endpoints of $D)$. Due to $\left|g^{\prime}(x)\right| \geq \nu-\varepsilon, g^{\prime} \in C(\bar{D})$, the length of each subinterval is majorized by

$$
\frac{2 \varepsilon}{\nu-\varepsilon}
$$

The result follows with $C=4(\widehat{n}+2) / \nu$ for $\varepsilon<\frac{\nu}{2}$.

Based on Propositions 4.1 - 4.3, we establish error estimates between the solutions of the problems (1.4), (1.5) and (3.6), (3.7).

Theorem 4.4. Let $y_{\varepsilon} \in H^{1}(D)$ be the solution of (3.7) and $y \in H^{1}(D)$ the solution of (1.5), where $\chi_{1}=H\left(p_{1}\right), \chi_{2}=H\left(p_{2}\right), \chi_{3}=1-H\left(p_{1}\right)-H\left(p_{2}\right)$, with $\left[p_{1}, p_{2}\right] \in H_{\mathrm{loc}}^{1}(D)^{2}$ satisfying (3.1), (3.3). 
Then we have

$$
\left|y_{\varepsilon}-y\right|_{H^{1}(D)} \leq C \mu\left(D_{\varepsilon}\right)^{q},
$$

with $q>0$ depending on the dimension of $D, D_{\varepsilon}$ explained below, and $C>0$ independent of $\varepsilon>0$.

Proof. We subtract the equations (1.5), (3.7) and use the test function $v=$ $\left(y-y_{\varepsilon}\right)$ :

$$
\begin{aligned}
0=\int_{D}\{ & {\left[a_{1} H\left(p_{1}\right)+a_{2} H\left(p_{2}\right)+\left(1-H\left(p_{1}\right)-H\left(p_{2}\right)\right) a_{3}\right]\left|\nabla\left(y-y_{\varepsilon}\right)\right|^{2} } \\
& \left.\quad+a_{0}\left|y-y_{\varepsilon}\right|^{2}\right\} \mathrm{d} x \\
+ & \int_{D}\left[\left(a_{1}-a_{3}\right)\left(H\left(p_{1}\right)-H_{\varepsilon}\left(p_{1}\right)\right)\right. \\
& \left.\quad+\left(a_{2}-a_{3}\right)\left(H\left(p_{2}\right)-H_{\varepsilon}\left(p_{2}\right)\right)\right] \nabla y_{\varepsilon} \cdot \nabla\left(y-y_{\varepsilon}\right) \mathrm{d} x
\end{aligned}
$$

The last integral in (4.3) is in fact over $D_{\varepsilon}\left(p_{1}\right) \cup D_{\varepsilon}\left(p_{2}\right)=: D_{\varepsilon}$ (new notation).

By the assumption on $a_{0}, \ldots, a_{3}$, it yields

$$
\left|y-y_{\varepsilon}\right|_{H^{1}(D)}^{2} \leq C \int_{D_{\varepsilon}}\left|\nabla y_{\varepsilon}\right|\left|\nabla\left(y_{\varepsilon}-y\right)\right| \mathrm{d} x,
$$

where $C$ is computed from the coefficients and is independent of $\varepsilon>0$. Applying the Cauchy-Schwarz and the Hölder inequality in (4.4), we infer

$$
\begin{aligned}
\left|y_{\varepsilon}-y\right|_{H^{1}(D)} & \leq C\left(\int_{D_{\varepsilon}}\left|\nabla y_{\varepsilon}\right|^{2} \mathrm{~d} x\right)^{\frac{1}{2}} \\
& \leq C\left(\int_{D_{\varepsilon}}\left|\nabla y_{\varepsilon}\right|^{S} \mathrm{~d} x\right)^{\frac{1}{S}} \cdot \mu\left(D_{\varepsilon}\right)^{\frac{S-2}{S}} \leq C_{1} \mu\left(D_{\varepsilon}\right)^{\frac{S-2}{S}} .
\end{aligned}
$$

In (4.5), $S>2$ is given by the regularity and the boundedness in $W^{1, S}(D)$ of the solution of (3.7), Meyers [14]. The proof is finished with $q=\frac{S-2}{S}>$ 0 . 
We also indicate a direct argument in dimension one (which is of special interest here) of the regularity result for (3.7) (similarly for (1.5)).

Lemma 4.5. If $\operatorname{dim} D=1$, then $y_{\varepsilon}$, the solution of (3.7), satisfies $y_{\varepsilon} \in$ $W^{1, p}(D)$ for each $1 \leq p<\infty$, and $\left|y_{\varepsilon}^{\prime}\right|_{L^{p}(D)} \leq C|f|_{L^{2}(D)}, C>0$ independent of $\varepsilon>0$. The estimate in Thm. 4.4 is valid for each $0<q<1$.

Proof. By the uniform ellipticity, the weak solution $y_{\varepsilon} \in H^{1}(D)$ of $(3.7)$ satisfies

$$
\left|y_{\varepsilon}\right|_{H^{1}(D)} \leq \widetilde{C}|f|_{L^{2}(D)}
$$

with $\widetilde{C}>0$ independent of $\varepsilon>0$ (and in arbitrary dimension).

We write (3.7) in the form

$$
\int_{D} a_{\varepsilon}(x) y_{\varepsilon}^{\prime} v^{\prime} \mathrm{d} x=\int_{D}\left[f v-a_{0} y_{\varepsilon} v\right] \mathrm{d} x,
$$

where $a_{\varepsilon} \in L^{\infty}(D), m \geq a_{\varepsilon}(x) \geq a>0$, is the whole coefficient appearing in the first term in (3.7).

Take in (4.7) $v_{n} \in H^{1}(D), v_{n} \rightarrow \int_{\alpha}^{x} w$, for some $\alpha \in D$, converging strongly in $W^{1, p^{\prime}}(D)$ with $w \in L^{p^{\prime}}(D)$ arbitrarily fixed, $\frac{1}{p}+\frac{1}{p^{\prime}}=1$. The right-hand side in (4.7) has a limit for $n \rightarrow \infty$ and the same is valid for the left-hand side. Consequently, $y_{\varepsilon}^{\prime}$ defines a linear bounded functional on $L^{p^{\prime}}(D)$ :

$$
\begin{aligned}
& \left|\int_{D} y_{\varepsilon}^{\prime} a_{\varepsilon}(x) w \mathrm{~d} x\right|=\left|\int_{D}\left[f \int_{\alpha}^{x} w-a_{0} y_{\varepsilon} \int_{\alpha}^{x} w\right] \mathrm{d} x\right| \\
& \leq\left[\int_{D}\left|f(x) \mu(D)^{\frac{1}{p}}\right| \mathrm{d} x+\int_{D}\left|a_{0} y_{\varepsilon}(x) \mu(D)^{\frac{1}{p}}\right| \mathrm{d} x\right]|w|_{L^{p^{\prime}}(D)}
\end{aligned}
$$


by the Hölder inequality. By the known bounds for $a_{\varepsilon}(\cdot)$ and the boundedness of $\left\{y_{\varepsilon}\right\}$ in $H^{1}(D)$ as a weak solution according to (4.6), we get that $y_{\varepsilon}^{\prime} \in$ $L^{p}(D), \forall p<\infty$.

Corollary 4.6. For the minimal values of the problems $(R)$ and $\left(R_{\varepsilon}\right)$, we have the estimate

$$
\left|\min (R)-\min \left(R_{\varepsilon}\right)\right| \leq \widetilde{C} \mu\left(D_{\varepsilon}\right)^{q}
$$

with $\widetilde{C}>0$ independent of $\varepsilon>0$.

Proof. By the approximation property proved in Thm. 4.4, we obtain (see $(1.4))$ :

$$
\min (R) \geq \min \left(R_{\varepsilon}\right)-\widetilde{C} \mu\left(D_{\varepsilon}\right)^{q} .
$$

Conversely, if $\left[p_{1 \varepsilon}^{*}, p_{2 \varepsilon}^{*}\right]$ is an optimal pair for $\left(R_{\varepsilon}\right)$ and we introduce it in (1.5), then the estimate from Thm. 4.4 remains valid for the difference between the two states. Therefore, the converse inequality

$$
\min \left(R_{\varepsilon}\right) \geq \min (R)-\widetilde{C} \mu\left(D_{\varepsilon}\right)^{q}
$$

is valid and the proof is finished.

Remark. If we introduce $\left[p_{1 \varepsilon}^{*}, p_{2 \varepsilon}^{*}\right]$ in (1.5) and denote the obtained solution by $\widetilde{y}_{\varepsilon}$, then, by Thm. 4.4, we get

$$
|| \widetilde{y}_{\varepsilon}-\left.y_{d}\right|_{L^{2}(D)}-\left|y_{\varepsilon}^{*}-y_{d}\right|_{L^{2}(D)}|\leq| \widetilde{y}_{\varepsilon}-\left.y_{\varepsilon}^{*}\right|_{H^{1}(D)} \leq C \mu\left(D_{\varepsilon}\right)^{q},
$$

with $C>0$ independent of $\varepsilon>0$. Combining (4.8) with Corollary 4.6, we see that the cost obtained in $(R)$ from $\left[p_{1 \varepsilon}^{*}, p_{2 \varepsilon}^{*}\right]$, denoted by $R\left(p_{1 \varepsilon}^{*}, p_{2 \varepsilon}^{*}\right)$, satisfies

$$
\left|\min (R)-R\left(p_{1 \varepsilon}^{*}, p_{2 \varepsilon}^{*}\right)\right| \leq \check{C} \mu\left(D_{\varepsilon}\right)^{q} .
$$

This shows that $\left[p_{1 \varepsilon}^{*}, p_{2 \varepsilon}^{*}\right]$ is suboptimal in the original problem $(R)$. The subregions of $D$ corresponding to $H\left(p_{1 \varepsilon}^{*}\right), H\left(p_{2 \varepsilon}^{*}\right)$, and $\left(1-H\left(p_{1 \varepsilon}^{*}\right)-H\left(p_{2 \varepsilon}^{*}\right)\right)$ 
produce a cost at distance at most $\check{C} \mu\left(D_{\varepsilon}\right)^{q}$ from the optimal value, where $\check{C}>0$ is independent of $\varepsilon>0$.

Remark. In dimension 1, by Proposition 4.3, we obtain explicit estimates with respect to $\varepsilon>0$.

Remark. In the case of the problem (1.1) - (1.3), we have the estimate (2.6). By multiplying $(2.2),(2.3)$ by $\operatorname{sgn}\left(y_{\varepsilon}\right)$ (or a regularization $\operatorname{sgn}_{\lambda}\left(y_{\varepsilon}\right)$ ), one may infer

$$
\int_{D}\left(1-H_{\varepsilon}\left(g_{\varepsilon}^{*}\right)\right)\left|y_{\varepsilon}^{*}\right| \mathrm{d} x \leq C \varepsilon
$$

with $C>0$ independent of $\varepsilon>0$. This is due to the monotonicity of $\operatorname{sgn}(\cdot)$. However, both (2.6) or (4.9) seem too weak in order to extend the estimates of this section to the problem (1.1) - (1.3).

\section{Discretization}

Based on the results from previous sections, we study the discretization of the problems $\left(P_{\varepsilon}\right)$ and $\left(R_{\varepsilon}\right)$.

Notice that $\left(P_{\varepsilon}\right)$ is an optimal control problem defined in $D$ with the control $g$ acting in the coefficients of the lower order term. In this section, we assume

$$
g \in \widetilde{U}_{\mathrm{ad}}=\left\{z \in W^{1, \infty}(D):\left.z\right|_{E} \geq 0,|\nabla z|_{\mathbb{R}^{d}} \leq 1\right\}
$$

This is a variant of the conditions imposed on $g$ in Section 2 and it is partially justified by Thm. 2.2.

In $D$, we consider a uniformly regular finite element partition $D=$

$\bigcup_{T_{h} \in \mathcal{T}_{h}} T_{h}, h>0$. We assume that the grid in $D$, restricted to $E$, provides a finite element mesh in $E$ as well. 
Let $y_{\varepsilon} \in H^{2}(D) \cap H_{0}^{1}(D)$ be the unique solution of (2.2), (2.3). If $\operatorname{dim} D=$ $d \leq 3$, then $y_{\varepsilon} \in C(\bar{D})$, while (5.1) ensures $\widetilde{U}_{\text {ad }} \subseteq C(\bar{D})$ as well. An example when such properties hold is when $D$ is polyhedral and convex.

The discretization of $\left(P_{\varepsilon}\right)$ is the following

$$
\min _{g_{h} \in \widetilde{U}_{\text {ad }}^{h}} \int_{E} j\left(x, y_{\varepsilon, h}(x)\right) \mathrm{d} x,
$$

$$
\begin{gathered}
\int_{D} \nabla y_{\varepsilon, h} \cdot \nabla v_{h} \mathrm{~d} x+\frac{1}{\varepsilon} \int_{D}\left(1-H_{\varepsilon}\left(g_{h}\right)\right) y_{\varepsilon, h} v_{h} \mathrm{~d} x=\int_{D} f_{h} v_{h} \mathrm{~d} x, \\
\forall v_{h} \in V_{h} \subseteq H_{0}^{1}(D),
\end{gathered}
$$

$$
g_{h} \in \widetilde{U}_{\mathrm{ad}}^{h}=\left\{z_{h} \in \widetilde{V}_{h} \subseteq H^{1}(D):\left.z_{h}\right|_{E} \geq 0,\left|\nabla z_{h}\right|_{\mathbb{R}^{d}} \leq 1\right\}
$$

Here $V_{h}, \widetilde{V}_{h}$ are the finite element spaces in $D$ constructed with piecewise linear continuous functions (with 0 trace on $\partial D$ for elements of $V_{h}$ ) and $f_{h}$ is some interpolant of $f \in L^{2}(D), f_{h} \rightarrow f$ in $L^{2}(D)$. We denote simply by $\left[y^{h}, g^{h}\right] \in V_{h} \times \widetilde{U}_{\text {ad }}^{h}\left(\varepsilon\right.$ is fixed here) an optimal pair for $\left(P_{\varepsilon}^{h}\right)$. Its existence follows by the convexity and compactness properties of $\widetilde{U}_{\text {ad }}^{h}$ and the continuity properties of the mapping $g_{h} \rightarrow y_{\varepsilon, h}$ defined in (5.2).

Theorem 5.1. Assume that (5.1) is included in the definition of $\left(P_{\varepsilon}\right)$. Then, $\left[y^{h}, g^{h}\right] \rightarrow\left[y_{\varepsilon}, g_{\varepsilon}\right]$ in $H_{0}^{1}(D) \times C(\bar{D})$, on a subsequence for $h \rightarrow 0$, where $\left[y_{\varepsilon}, g_{\varepsilon}\right]$ is an optimal pair of $\left(P_{\varepsilon}\right)$.

Proof. On a subsequence, we may assume $g^{h} \rightarrow g_{\varepsilon} \in \widetilde{U}_{\text {ad }}$, strongly in $C(\bar{D})$. Fix $v_{h}=y^{h}$ in (5.2), corresponding to $g^{h}$. We get $\left\{y^{h}\right\}$ bounded in $H_{0}^{1}(D)$, where we also use $f_{h} \rightarrow f$ in $L^{2}(D)$.

On a subsequence, $y^{h} \rightarrow y_{\varepsilon}$ weakly in $H_{0}^{1}(D)$. For any $v \in H^{2}(D) \cap$ $H_{0}^{1}(D)$, one can find $v_{h} \in V_{h}$, with $v_{h} \rightarrow v$ strongly in $H_{0}^{1}(D)$. Then, it is 
possible to pass to the limit $h \rightarrow 0$ in the discretized state system (5.2) and to infer

$$
\int_{D} \nabla y_{\varepsilon} \cdot \nabla v \mathrm{~d} x+\frac{1}{\varepsilon} \int_{D}\left(1-H_{\varepsilon}\left(g_{\varepsilon}\right)\right) y_{\varepsilon} v \mathrm{~d} x=\int_{D} f v \mathrm{~d} x, \quad \forall v \in H_{0}^{1}(D) .
$$

In (5.4), we also use the density of $H^{2}(D)$ in $H^{1}(D)$. We obtain as well:

$$
\int_{E} j\left(x, y_{\varepsilon}(x)\right) \mathrm{d} x=\lim _{h \rightarrow 0} \int_{E} j\left(x, y^{h}(x)\right) \mathrm{d} x
$$

by the assumptions on $j(\cdot, \cdot)$ (see Thm. 2.2).

For any $g \in \widetilde{U}_{\mathrm{ad}}$, there is $g_{h} \in \widetilde{U}_{\mathrm{ad}}^{h}$ such that $g_{h} \rightarrow g$ in $C(\bar{D})$. If $y_{\varepsilon, h}$ denotes the solution of (5.2) corresponding to $g_{h}$, a similar argument shows that $y_{\varepsilon, h} \rightarrow \widetilde{y}_{\varepsilon}$ weakly in $H_{0}^{1}(D)$ and $\left[\widetilde{y}_{\varepsilon}, g\right]$ satisfy (5.4). As in (5.5), we get

$$
\int_{E} j\left(x, \widetilde{y}_{\varepsilon}(x)\right) \mathrm{d} x=\lim _{h \rightarrow 0} \int_{E} j\left(x, y_{\varepsilon, h}(x)\right) \mathrm{d} x .
$$

Taking into account (5.5), (5.6) and the optimality of $\left[y^{h}, g^{h}\right]$, we see that $\left[y_{\varepsilon}, g_{\varepsilon}\right]$ is an optimal pair for $\left(P_{\varepsilon}\right)$ and the proof is finished.

Remark. It is possible to prove a similar result for $U_{\text {ad }}$ as in Theorem 2.2. The advantage, here, is that $\widetilde{U}_{\text {ad }}, \widetilde{U}_{\text {ad }}^{h}$ are convex as well.

Theorem 5.2. The mapping $\theta_{\varepsilon, h}: \widetilde{V}_{h} \rightarrow V_{h}$, given by $g_{h} \mapsto y_{\varepsilon, h}$, is Gâteaux differentiable and $z_{h}=\nabla \theta_{\varepsilon, h}\left(g_{h}\right) w_{h}$ satisfies

$$
\int_{D} \nabla z_{h} \cdot \nabla v_{h} \mathrm{~d} x+\frac{1}{\varepsilon} \int_{D}\left(1-H_{\varepsilon}\left(g_{h}\right)\right) z_{h} v_{h} \mathrm{~d} x=\frac{1}{\varepsilon} \int_{D} H_{\varepsilon}^{\prime}\left(g_{h}\right) y_{\varepsilon, h} w_{h} v_{h} \mathrm{~d} x
$$

for any $v_{h} \in V_{h}$ and with $w_{h} \in \widetilde{V}_{h}$. 
Proof. Let $y_{\varepsilon, h}^{\lambda}=\theta_{\varepsilon, h}\left(g_{h}+\lambda w_{h}\right), \lambda \in \mathbb{R}$. Subtract the equations corresponding to $y_{\varepsilon, h}^{\lambda}, y_{\varepsilon, h}$ and divide by $\lambda \neq 0$ :

$$
\begin{aligned}
& \int_{D}\left[\nabla \frac{y_{\varepsilon, h}^{\lambda}-y_{\varepsilon, h}}{\lambda} \cdot \nabla v_{h}+\frac{1}{\varepsilon}\left(1-H_{\varepsilon}\left(g_{h}+\lambda w_{h}\right)\right) \frac{y_{\varepsilon, h}^{\lambda}-y_{\varepsilon, h}}{\lambda} v_{h}\right] \mathrm{d} x \\
& =\frac{1}{\varepsilon} \int_{D} \frac{H_{\varepsilon}\left(g_{h}+\lambda w_{h}\right)-H_{\varepsilon}\left(g_{h}\right)}{\lambda} y_{\varepsilon, h} v_{h} \mathrm{~d} x, \quad \forall v_{h} \in V_{h} .
\end{aligned}
$$

Since $H_{\varepsilon}(\cdot)$ is in $C^{1}(\bar{D})$ and $g_{h}+\lambda w_{h} \rightarrow g_{h}$ in $C(\bar{D})$ for $\lambda \rightarrow 0$, we have

$$
\frac{H_{\varepsilon}\left(g_{h}+\lambda w_{h}\right)-H_{\varepsilon}\left(g_{h}\right)}{\lambda} \rightarrow H_{\varepsilon}^{\prime}\left(g_{h}\right) w_{h}
$$

uniformly in $\bar{D}$. Taking $v_{h}=\frac{y_{\varepsilon, h}^{\lambda}-y_{\varepsilon, h}}{\lambda}$ in (5.8), we see that it is bounded in $H_{0}^{1}(D)$ and in $V_{h}$ with respect to $\lambda \rightarrow 0$ ( $\varepsilon$ and $h$ are fixed here). Denoting by $z_{h}$ its weak limit in $H_{0}^{1}(D)$ and in $V_{h}$ on a subsequence, we obtain (5.7), for $\lambda \rightarrow 0$. The limit is in fact in the strong topology since $V_{h}$ is finite dimensional. As the solution of (5.7) is unique and $z_{h}$ depends linearly and continuously on $w_{h}$, the proof is finished since the convergence is in fact valid without taking subsequences.

Under the assumption that $j_{y}\left(x, y_{h}\right)$ exists and belongs to $L^{2}(E)$ for each $y_{h} \in V_{h}$, we define the so-called adjoint system for the adjoint state $p_{h} \in V_{h}$ :

$$
\begin{aligned}
& \int_{D}\left[\nabla p_{h} \cdot \nabla v_{h}+\frac{1}{\varepsilon}\left(1-H_{\varepsilon}\left(g_{h}\right)\right) p_{h} v_{h}\right] \mathrm{d} x \\
& =\int_{E} j_{y}\left(x, y_{\varepsilon, h}\right) v_{h} \mathrm{~d} x, \quad \forall v_{h} \in V_{h} .
\end{aligned}
$$

Corollary 5.3. The directional derivative of the cost in $\left(P_{\varepsilon}^{h}\right)$, at $g_{h} \in \widetilde{V}_{h}$ and in the direction $w_{h} \in \widetilde{V}_{h}$, is given by

$$
\frac{1}{\varepsilon} \int_{D} H_{\varepsilon}^{\prime}\left(g_{h}\right) y_{\varepsilon, h} w_{h} p_{h} \mathrm{~d} x,
$$


where $p_{h} \in V_{h}$ is given by (5.9).

Proof. We compute the limit

$$
\begin{aligned}
& \lim _{\lambda \rightarrow 0} \frac{1}{\lambda}\left[\int_{E} j\left(x, y_{\varepsilon, h}^{\lambda}\right) \mathrm{d} x-\int_{E} j\left(x, y_{\varepsilon, h}\right) \mathrm{d} x\right] \\
& =\int_{D}\left[\nabla p_{h} \cdot \nabla z_{h}+\frac{1}{\varepsilon}\left(1-H_{\varepsilon}\left(g_{h}\right)\right) p_{h} z_{h}\right] \mathrm{d} x=\frac{1}{\varepsilon} \int_{D} H_{\varepsilon}^{\prime}\left(g_{h}\right) y_{\varepsilon, h} w_{h} p_{h} \mathrm{~d} x
\end{aligned}
$$

by using (5.9), (5.7), under the notations of Theorem 5.2.

Remark. By Corollary 5.3, we notice the descent directions $\widetilde{w}_{h}=-\frac{1}{\varepsilon} H_{\varepsilon}^{\prime}\left(g_{h}\right) y_{\varepsilon, h} p_{h}$ and $\widehat{w}_{h}=-y_{\varepsilon, h} p_{h}$ (since the coefficient is positive). Notice that the support of $H_{\varepsilon}^{\prime}\left(g_{h}\right)$ is contained in $E_{\varepsilon}\left(g_{h}\right)$ (see (4.2)). By (1.6), this is a neighborhood of the boundary of $\Omega_{g_{h}}$. Therefore, roughly speaking, $\widetilde{w}_{h}$ corresponds to boundary variations if gradient methods are applied in $(P)$. The descent direction $\widehat{w}_{h}$ is more general and allows simultaneous boundary and topological variations, which is useful in applications. Remark. For cost functionals of the type $\int_{\Omega} j(x, y(x)) \mathrm{d} x$ and their approximation (2.5) in $D$, one has to put $\int_{D} H_{\varepsilon}(g) j_{y}\left(x, y_{\varepsilon, h}\right) v_{h} \mathrm{~d} x$ in the right-hand side of (5.9).

For the problem $\left(R_{\varepsilon}\right)$, due to $(3.1)$ - (3.3) defining $U_{\text {ad }}$, it is necessary to use higher order finite elements for the discretization of $\left[p_{1}, p_{2}\right] \in U_{\mathrm{ad}}$.

We define $U_{\text {ad }}^{h}$ by the conditions:

$$
\begin{gathered}
\left|p_{i}^{h}(x)\right|+\left|\nabla p_{i}^{h}(x)\right|_{\mathbb{R}^{d}} \geq \nu>0 \quad \text { a.e. } D, i=1,2, \\
\left|p_{i}^{h}\right|_{H^{2}(K)} \leq M_{K}^{i}, i=1,2
\end{gathered}
$$

for each $K \subseteq D$ compactly embedded open subset, 


$$
p_{i}^{h}(x) \geq 0 \quad \Rightarrow \quad p_{j}^{h}(x) \leq 0 .
$$

This is a slight strengthening of (3.1) - (3.3) and $U_{\mathrm{ad}}^{h} \subseteq H_{\mathrm{loc}}^{2}(D)^{2}$.

For the approximation of the solution of the equation (3.7), piecewise linear finite elements are used, denoted by $V_{h} \subseteq H^{1}(D)$.

The discretized optimization problem is $\left(R_{\varepsilon}^{h}\right)$

$$
\begin{gathered}
\min _{\left[p_{1}^{h}, p_{2}^{h}\right] \in U_{\mathrm{ad}}^{h}} \int_{E}\left|y_{h}-y_{d}\right|^{2} \mathrm{~d} x \\
\int_{D}\left\{\left[a_{1} H_{\varepsilon}\left(p_{1}^{h}\right)+a_{2} H_{\varepsilon}\left(p_{2}^{h}\right)+a_{3}\left(1-H_{\varepsilon}\left(p_{1}^{h}\right)-H_{\varepsilon}\left(p_{2}^{h}\right)\right)\right] \nabla y_{h} \cdot \nabla v_{h}\right. \\
\left.+a_{0} y_{h} v_{h}-f v_{h}\right\} \mathrm{d} x=0, \quad \forall v_{h} \in V_{h} .
\end{gathered}
$$

For simplicity, no discretization of $a_{i}, i=\overline{0,3}, f$ and $y_{d}$ is performed. The finite element mesh in $D, E$ is as in $\left(P_{\varepsilon}^{h}\right)$.

Theorem 5.4. The problem $\left(R_{\varepsilon}^{h}\right)$ has at least one optimal triple denoted $\left[y_{h}^{*}, p_{1 h}^{*}, p_{2 h}^{*}\right] \in V_{h} \times U_{\mathrm{ad}}^{h}$. On a subsequence $h \rightarrow 0$, we have

$$
\begin{aligned}
& y_{h}^{*} \rightarrow y_{\varepsilon}^{*} \quad \text { weakly in } H^{1}(D), \\
& p_{1 h}^{*} \rightarrow p_{1 \varepsilon}^{*} \quad \text { in } H_{\mathrm{loc}}^{1}(D), \\
& p_{2 h}^{*} \rightarrow p_{2 \varepsilon}^{*} \quad \text { in } H_{\mathrm{loc}}^{1}(D),
\end{aligned}
$$

where $\left[y_{h}^{*}, p_{1 h}^{*}, p_{2 h}^{*}\right]$ is an optimal triple for $\left(R_{\varepsilon}\right)$, under assumption $\theta_{k}^{i}=1$, $\forall K \subseteq D$ compactly embedded, $i=1,2$, in (3.2).

Sketch of Proof. The existence of $\left[y_{h}^{*}, p_{1 h}^{*}, p_{2 h}^{*}\right]$, for $h>0$ fixed, follows as in $\S 3$, using the boundedness of $U_{\mathrm{ad}}^{h}$ in $H_{\mathrm{loc}}^{2}(D)^{2}$. Moreover $p_{i h}^{*} \rightarrow p_{i \varepsilon}^{*}, i=1,2$, on a subsequence, in $H_{\mathrm{loc}}^{1}(D)$ by the Sobolev theorem. We get $\left[p_{1 \varepsilon}^{*}, p_{2 \varepsilon}^{*}\right] \in U_{\mathrm{ad}}$ (where $\theta_{k}^{i}=1, i=1,2$ in $(3.2)$ ). 
We get $\left\{y_{h}^{*}\right\}$ bounded in $H^{1}(D)$ by fixing $v_{h}=y_{h}^{*}$ in (5.14) written for $\left[p_{1 h}^{*}, p_{2 h}^{*}\right]$. On a subsequence, $y_{h}^{*} \rightarrow y_{\varepsilon}^{*}$ weakly in $H^{1}(D)$.

It is possible to pass to the limit in (5.13), (5.14) as in Section 3, to prove the optimality of $\left[y_{\varepsilon}^{*}, p_{1 \varepsilon}^{*}, p_{2 \varepsilon}^{*}\right]$ for $\left(R_{\varepsilon}\right)$.

Remark. It is also possible to take simultaneously $\varepsilon \rightarrow 0, h \rightarrow 0$ to show the approximation of $(R)$. In the one dimensional case, for two materials, rates of convergence are established in [4].

Remark. Concerning condition (3.1), in dimension one, it is to be noticed that it is automatically satisfied by trigonometric functions, which may provide another advantageous approximation.

\section{$6 \quad$ Numerical Experiments}

The following numerical experiments were conducted in a setting that combines aspects of $(1.1)-(1.3)$ with aspects of (1.4), (1.5), namely

$$
\begin{aligned}
& \text { (6.1) } \quad \min _{\Omega, \chi} \frac{1}{2} \int_{E}\left|y-y_{d}\right|^{2} \mathrm{~d} x+\frac{\sigma}{2} \int_{E}\left|\nabla y-\nabla y_{d}\right|^{2} \mathrm{~d} x \\
& \quad \int_{\Omega}\left[a_{1} \chi+a_{2}(1-\chi)\right] \nabla y \cdot \nabla v \mathrm{~d} x+\int_{\Omega}\left[b_{1} \chi+b_{2}(1-\chi)\right] y v \mathrm{~d} x \\
& (6.2) \quad=\int_{\Omega} f v \mathrm{~d} x, \quad \forall v \in H_{0}^{1}(\Omega) \\
& \text { (6.3) } \quad y-\xi \in H_{0}^{1}(\Omega)
\end{aligned}
$$

where $E \subseteq O \subseteq \Omega \subseteq D \subseteq \mathbb{R}^{2} ; E, D$ being given and fixed as before; $\Omega, O$ to be optimized with $\chi$ denoting the characteristic function of $O ; \sigma \geq 0$, $a_{1}, a_{2}, b_{1}, b_{2}>0, f \in L^{2}(D), \xi \in H^{1}(D)$ all given. As explained in [15], (6.1) - (6.3) are motivated by the oil industry application studied in [29]. The 
regularized fixed-domain version of (6.2), (6.3) in discretized form is

$$
\begin{aligned}
& \int_{D}\left[a_{1} H_{\varepsilon}\left(p_{h}\right)+a_{2}\left(1-H_{\varepsilon}\left(p_{h}\right)\right)\right] \nabla y_{\varepsilon, h} \cdot \nabla v_{h} \mathrm{~d} x \\
& \quad+\int_{D}\left[b_{1} H_{\varepsilon}\left(p_{h}\right)+b_{2}\left(1-H_{\varepsilon}\left(p_{h}\right)\right)\right] y_{\varepsilon, h} v_{h} \mathrm{~d} x \\
& \quad+\frac{1}{\varepsilon} \int_{D}\left(1-H_{\varepsilon}\left(g_{h}\right)\right) y_{\varepsilon, h} v_{h} \mathrm{~d} x \\
& =\int_{D} f_{h} v_{h} \mathrm{~d} x, \quad \forall v_{h} \in V_{h} \subseteq H_{0}^{1}(D), \\
& y_{\varepsilon, h}-\xi_{h} \in V_{h} \subseteq H_{0}^{1}(D),
\end{aligned}
$$

yielding the equation for the discretized state $y_{\varepsilon, h} \in \widetilde{V}_{h} \subseteq H^{1}(D)$, where $f_{h} \in \widetilde{V}_{h}, f_{h} \rightarrow f$ in $L^{2}(D)$, and $\xi_{h} \in \widetilde{V}_{h}, \xi_{h} \rightarrow \xi$ in $H^{1}(D)$ are given; $g_{h}, p_{h} \in \widetilde{V}_{h}$ are discretized shape functions corresponding to discretizations of $\Omega$ and $O$, respectively; and $V_{h}, \widetilde{V}_{h}$ are as is in the first part of Section 5, i.e. finite element spaces in $D$ constructed with piecewise linear continuous functions (with 0 trace on $\partial D$ for elements of $V_{h}$ ). In particular, depending on $\xi$, (6.5) can mean homogeneous or nonhomogeneous Dirichlet conditions. The shape optimization for (6.4), (6.5) takes the form

$$
\begin{aligned}
& \min _{\left(g_{h}, p_{h}\right) \in U_{\mathrm{ad}}^{h}} j\left(g_{h}, p_{h}\right):= \frac{1}{2} \int_{E}\left|y_{\varepsilon, h}-y_{d, h}\right|^{2} \mathrm{~d} x \\
&+\frac{\sigma}{2} \int_{E}\left|\nabla y_{\varepsilon, h}-\nabla y_{d, h}\right|^{2} \mathrm{~d} x, \\
& U_{\mathrm{ad}}^{h}:=\left\{[g, p] \in \widetilde{V}_{h} \times \widetilde{V}_{h}: g \geq p \text { on } D \text { and } p \geq 0 \text { on } E\right\},
\end{aligned}
$$

with given $y_{d, h} \rightarrow y_{d}$ in $H^{1}(D)$.

Then one obtains the discretized adjoint equation for the discretized ad- 
joint state $q_{\varepsilon, h} \in V_{h} \subseteq H_{0}^{1}(D)$ (cf. [15, (34)]):

$$
\begin{aligned}
\int_{D}[ & \left.a_{1} H_{\varepsilon}\left(p_{h}\right)+a_{2}\left(1-H_{\varepsilon}\left(p_{h}\right)\right)\right] \nabla q_{\varepsilon, h} \cdot \nabla v_{h} \mathrm{~d} x \\
& +\int_{D}\left[b_{1} H_{\varepsilon}\left(p_{h}\right)+b_{2}\left(1-H_{\varepsilon}\left(p_{h}\right)\right)\right] q_{\varepsilon, h} v_{h} \mathrm{~d} x \\
& +\frac{1}{\varepsilon} \int_{D}\left(1-H_{\varepsilon}\left(g_{h}\right)\right) q_{\varepsilon, h} v_{h} \mathrm{~d} x \\
= & \int_{E}\left(y_{\varepsilon, h}-y_{d, h}\right) v_{h} \mathrm{~d} x+\sigma \int_{E}\left(\nabla y_{\varepsilon, h}-\nabla y_{d, h}\right) \cdot \nabla v_{h} \mathrm{~d} x, \quad \forall v_{h} \in V_{h},
\end{aligned}
$$

and the descent directions (cf. [15, Rem. 9] and the first part of Section 5 above)

$$
\begin{aligned}
w_{\mathrm{d}}\left(y_{\varepsilon, h}, q_{\varepsilon, h}\right) & :=-y_{\varepsilon, h} q_{\varepsilon, h}, \\
v_{\mathrm{d}}\left(y_{\varepsilon, h}, q_{\varepsilon, h}\right) & :=\left(a_{1}-a_{2}\right) \nabla y_{\varepsilon, h} \cdot \nabla q_{\varepsilon, h}+\left(b_{1}-b_{2}\right) y_{\varepsilon, h} q_{\varepsilon, h} .
\end{aligned}
$$

Based on the above, we formulate the algorithm employed to obtain the numerical results presented below. The algorithm is of gradient with projection type.

Algorithm 6.1. Step 1: Set $n:=0$ and choose admissible initial shape functions $\left[g_{h, 0}, p_{h, 0}\right] \in U_{\mathrm{ad}}^{h}$.

Step 2: Compute the solution to the state equation $y_{n}:=\theta_{\varepsilon, h}\left(g_{h, n}, p_{h, n}\right)$, where $\theta_{\varepsilon, h}: \widetilde{V}_{h} \times \widetilde{V}_{h} \longrightarrow \widetilde{V}_{h}$ denotes the control-to-state operator corresponding to $(6.4),(6.5)$.

Step 3: Compute the solution to the corresponding adjoint equation $q_{n}:=$ $\tilde{\theta}_{\varepsilon, h}\left(y_{n}\right)$, where $\tilde{\theta}_{\varepsilon, h}: \widetilde{V}_{h} \longrightarrow V_{h}, y_{\varepsilon, h} \mapsto q_{\varepsilon, h}$, denotes the solution operator corresponding to $(6.8)$.

Step 4: Compute descent directions $w_{\mathrm{d}, n}=w_{\mathrm{d}, n}\left(y_{n}, q_{n}\right), v_{\mathrm{d}, n}=v_{\mathrm{d}, n}\left(y_{n}, q_{n}\right)$ according to (6.9). 
Step 5: Set $\tilde{g}_{n}:=g_{n}+\lambda_{n} w_{\mathrm{d}, n}$ and $\tilde{p}_{n}:=p_{n}+\lambda_{n} v_{\mathrm{d}, n}$, where $\lambda_{n} \geq 0$ is determined via line search, i.e. as a solution to the minimization problem

$$
\min _{\lambda \geq 0} j\left(g_{n}+\lambda w_{\mathrm{d}, n}, p_{n}+\lambda v_{\mathrm{d}, n}\right)
$$

Step 6: Set $\left(g_{n+1}, p_{n+1}\right):=\pi_{h}\left(\tilde{g}_{n}, \tilde{p}_{n}\right)$, where $\pi_{h}$ denotes the projection

$$
\pi_{h}: \widetilde{V}_{h} \times \widetilde{V}_{h} \longrightarrow U_{\mathrm{ad}}^{h}
$$

which is obtained by first setting $g_{n+1}\left(x_{i}^{h}\right):=\max \left\{0, \tilde{g}_{n}\left(x_{i}^{h}\right)\right\}$ and $\bar{p}_{n+1}\left(x_{i}^{h}\right):=\max \left\{0, \tilde{p}_{n}\left(x_{i}^{h}\right)\right\}$ for each node $x_{i}^{h}$ of the triangulation $\mathcal{T}_{h}$ such that $x_{i}^{h} \in \bar{E}$, and second setting

$$
p_{n+1}\left(x_{i}^{h}\right):=\min \left\{\bar{p}_{n+1}\left(x_{i}^{h}\right), g_{n+1}\left(x_{i}^{h}\right)\right\}
$$

for every node $x_{i}^{h}$ of the triangulation $\mathcal{T}_{h}$.

Step 7: RETURN $\left(g_{\text {fin }}, p_{\text {fin }}\right):=\left(g_{n+1}, p_{n+1}\right)$ if the change of $g, p$ and/or the change of $j(g, p)$ are below some prescribed tolerance parameter. Otherwise: Increment $n$, i.e. $n:=n+1$ and GO TO Step 2.

For all the numerical examples discussed subsequently, we stopped the iteration and returned $\left(g_{\text {fin }}, p_{\text {fin }}\right):=\left(g_{n+1}, p_{n+1}\right)$ if $\mid j\left(g_{n}, p_{n}\right)-$ $j\left(g_{n+1}, p_{n+1}\right) \mid<10^{-8}$ AND $\left\|g_{n}-g_{n+1}\right\|_{2}<10^{-3}$ AND $\left\|p_{n}-p_{n+1}\right\|_{2}<$ $10^{-3}$, where $\left|j\left(g_{n}, p_{n}\right)-j\left(g_{n+1}, p_{n+1}\right)\right| /\left|j\left(g_{n+1}, p_{n+1}\right)\right|$ is used instead of $\left|j\left(g_{n}, p_{n}\right)-j\left(g_{n+1}, p_{n+1}\right)\right|$ if $\left|j\left(g_{n+1}, p_{n+1}\right)\right|>1$ and analogous for $g_{n}$ and $p_{n}$.

The state equations as well as the adjoint equations that need to be solved numerically during the above algorithm are discretized linear elliptic PDE with Dirichlet boundary conditions. The numerical solution is obtained via 
a finite volume scheme [19, Sect. 4], [7, Chap. III]. More precisely, the software WIAS-HiTNIHS ${ }^{1}$, originally designed for the solution of more general PDE occurring when modeling conductive-radiative heat transfer and electromagnetic heating $[9,12]$, has been adapted for use in the present context. WIAS-HiTNIHS is based on the program package pdelib [8], it employs the grid generator Triangle $[24,25]$ to produce constrained Delaunay triangulations of the domains, and it uses the sparse matrix solver GSPAR [11] to solve the linear system arising from the finite volume scheme.

The numerical scheme yields discrete $y_{n}$ and $q_{n}$ (cf. Steps 2 and 3 of the above algorithm), defined at each vertex of the triangular discrete grid, interpolated piecewise affine, i.e. affinely to each triangle of the discrete grid. In consequence, the shape functions $g_{n}$ and $p_{n}$ are piecewise affine as well. Where integrals of these piecewise affine functions need to be computed (e.g. in Step 7 of the algorithm), they are computed exactly. A golden section search [22, Sect. 10.2] is used to numerically carry out the minimization (6.10). Note that the minimization (6.10) is typically nonconvex and the golden section search will, in general, only provide a local min $\lambda_{n}$.

For the regularized Heaviside mapping, we use

$$
H_{\varepsilon}(r):= \begin{cases}1 & \text { for } r \geq 0, \\ \frac{\varepsilon(r+\varepsilon)^{2}-2 r(r+\varepsilon)^{2}}{\varepsilon^{3}} & \text { for }-\varepsilon<r<0, \\ 0 & \text { for } r \leq-\varepsilon .\end{cases}
$$

Remark. For some of the following examples, the stated initial shape functions $[g, p]$ are merely piecewise continuous (cf. the Introduction and [20]) and, thus, not in $U_{\mathrm{ad}}^{h}$. However, the stated $[g, p]$ are only used to determine the values $g_{h}\left(x_{i}^{h}\right), p_{h}\left(x_{i}^{h}\right)$, at the nodes $x_{i}^{h}$ of the triangulation $\mathcal{T}_{h}$, and

\footnotetext{
${ }^{1}$ High Temperature Numerical Induction Heating Simulator; pronunciation: hitnice.
} 
$\left[g_{h}, p_{h}\right] \in U_{\text {ad }}^{h}$ for the resulting affinely interpolated $\left[g_{h}, p_{h}\right]$.

Example 6.2. The numerical computations of the present example employ the circular fixed domain

$$
D:=\left\{\left(x_{1}, x_{2}\right): x_{1}^{2}+x_{2}^{2}<1\right\} \subseteq \mathbb{R}^{2}
$$

with fixed subdomain

$$
E:=\left\{\left(x_{1}, x_{2}\right) \in D:\left|x_{1}\right|>\frac{3}{4},\left|x_{2}\right|<\frac{1}{2}\right\} \subseteq D
$$

(note that $E$ has two connected components).

We use a fixed triangular grid provided by Triangle [24, 25], consisting of 24458 triangles.

The sets determined by the shape functions $g, p$ are

$$
\begin{aligned}
& \Omega_{g}:=\operatorname{int}\{x \in D: g(x) \geq 0\} \\
& \Omega_{p}:=\operatorname{int}\{x \in D: p(x) \geq 0\} .
\end{aligned}
$$

Parameter settings:

$$
\begin{aligned}
& \varepsilon:=10^{-7}, \\
& a_{1}:=1, \quad a_{2}:=10, \quad b_{1}:=1, \quad b_{2}:=10 \\
& f\left(x_{1}, x_{2}\right):=5, \\
& \xi\left(x_{1}, x_{2}\right):=2 .
\end{aligned}
$$

The cost functional $j$ as in (6.6) depends on the given function $y_{d, h}$. For the first set of numerical results, we precompute $y_{d, h}:=y_{\varepsilon, h}$ numerically as the solution to the state equation (6.4), (6.5), using

$$
g\left(x_{1}, x_{2}\right):=1, \quad p\left(x_{1}, x_{2}\right):= \begin{cases}1 & \text { in } E \\ -1 & \text { in } D \backslash E .\end{cases}
$$



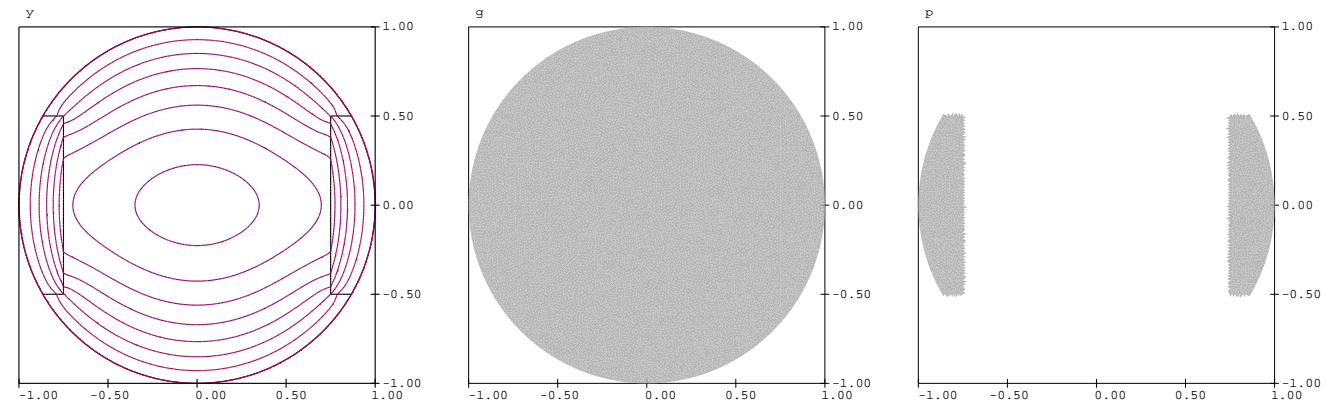

Figure 1: Precomputed $y_{d, h}$ used in Examples 6.2(a) - 6.2(c) (left, isolevels spaced at $0.05)$, obtained as the solution to the state equation (6.4), (6.5); depicted together with the corresponding $\Omega_{g}$ (middle, $g \geq 0$ in gray) and $\Omega_{p}$ (right, $p \geq 0$ in gray) - more precisely, the color of each triangle of the discretization is determined by the average of $g$ (resp. $p$ ) on that triangle.

The computed $y_{d, h}$ together with the corresponding $\Omega_{g}$ and $\Omega_{p}$ is depicted in Fig. 1. Using the precomputed $y_{d, h}$ has the advantage that we actually know $y_{d, h}$ together with $g, p$ as in (6.17) provides an absolute minimum in the following Examples (a) - (c).

(a) Setting $\sigma:=1$, the cost functional $j$ is

$$
j(g, p):=\frac{1}{2} \int_{E}\left|y_{\varepsilon, h}-y_{d, h}\right|^{2} \mathrm{~d} x+\frac{1}{2} \int_{E}\left|\nabla y_{\varepsilon, h}-\nabla y_{d, h}\right|^{2} \mathrm{~d} x
$$

with the precomputed $y_{d, h}$ from above. Since $j \geq 0$ and $j(g, p)=0$ for $g, p$ as in (6.17), these functions are optimal for $j$.

The initial shape functions are

$$
\begin{aligned}
& g_{0}\left(x_{1}, x_{2}\right):=1, \\
& p_{0}\left(x_{1}, x_{2}\right):=1 .
\end{aligned}
$$

Results are depicted in Fig. 2. In this example, we observe rapid convergence to the known minimum within two line searches, where the cost is reduced from the initial value of 1.18 to virtually 0 . The employed 
method is designed to facilitate topological changes of the shapes during the optimization process, and, in Fig. 1, we see that such changes, indeed, occur (as they must, in this case, for the minimum to be found). Also note that the intermediate shapes shown in Fig. 1 correspond to shape functions $[g, p] \notin U_{\mathrm{ad}}^{h}$. Such inadmissible shape functions are to be expected during line searches, where Step 6 of Algorithm 6.1 projects back into $U_{\text {ad }}^{h}$ after each line search. In a final note on Fig. 1, we observe that the radial symmetry of the initial condition is broken by $j$ (both $E$ and $y_{d, h}$ are not radially symmetric), only retaining the symmetries with respect to the $x_{1}$-axis and the $x_{2}$-axis.

The rapid convergence to the precomputed optimum observed in the present situation is actually not typical, as the problem is strongly nonconvex, and the algorithm will typically converge to some different local minimum determined by the initial condition (cf. Examples 6.2(b),(c) below).

(b) Setting $\sigma:=0$, the cost functional $j$ is

$$
j(g, p):=\frac{1}{2} \int_{E}\left|y_{\varepsilon, h}-y_{d, h}\right|^{2} \mathrm{~d} x
$$

with the precomputed $y_{d, h}$ from above. The initial shape functions are the same as in (6.19).

Results are depicted in Fig. 3. As compared to Example 6.2(a), the convergence is slower, the convergence criteria of Algorithm 6.1, Step 7 , only being satisfied after the fifth line search. Due to the absence of the gradient term in the cost functional, the initial cost is already much lower than in (a). The final state corresponds to a local min close, but not identical, to the precomputed absolute min. 

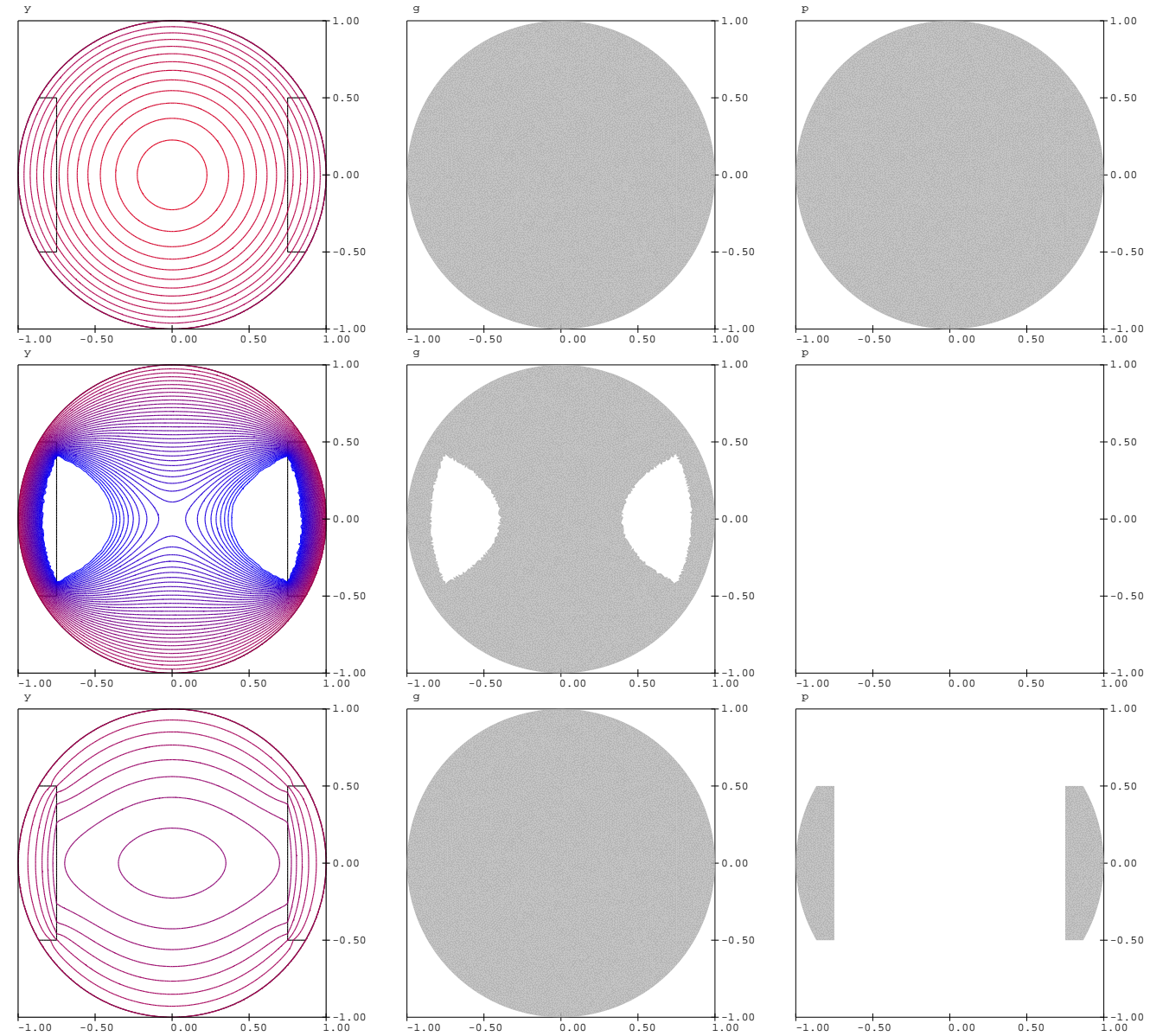

Figure 2: Shape optimization of Example 6.2(a). Left: State isolevels spaced at 0.05. Middle: Shapes $\Omega_{g}$ determined by $g \geq 0$ (in gray). Right: Shapes $\Omega_{p}$ determined by $p \geq 0$ (in gray). Row 1: initial state, shapes, $j\left(g_{0}, p_{0}\right)=1.18$. Row 2 : intermediate state, shapes during line search $\# 1$, cost $=0.102$. Row 3: final state, shapes (after 2 line searches, $j\left(g_{\mathrm{fin}}, p_{\mathrm{fin}}\right)=4.03 \cdot 10^{-27}$, precomputed optimal state of Fig. 1 has been recovered).

(c) Returning to $\sigma:=1$ and the cost functional $j$ as in (6.18), we now vary the initial condition, using

$$
\begin{aligned}
& g_{0}\left(x_{1}, x_{2}\right):= \begin{cases}1 & \text { if } x_{2} \leq 0.9, \\
-1 & \text { otherwise }\end{cases} \\
& p_{0}\left(x_{1}, x_{2}\right):=g_{0}\left(x_{1}, x_{2}\right) .
\end{aligned}
$$

Results are depicted in Fig. 4. Even though the initial shapes do not seem far from the ones used in Example 6.2(a), the present situation is 

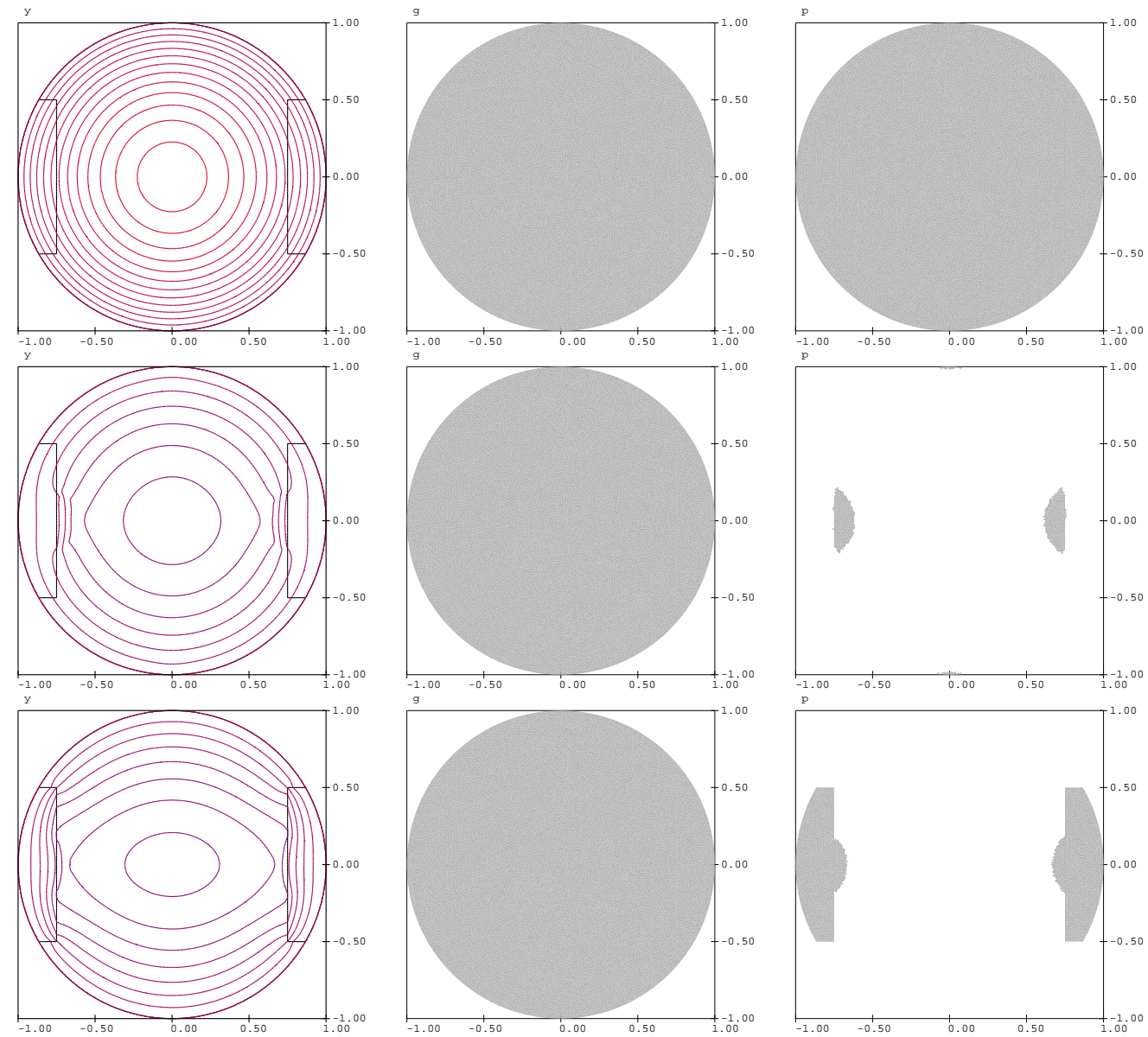

Figure 3: Shape optimization of Example 6.2(b). Left: State isolevels spaced at 0.05. Middle: Shapes $\Omega_{g}$ determined by $g \geq 0$ (in gray). Right: Shapes $\Omega_{p}$ determined by $p \geq 0$ (in gray). Row 1: initial state, shapes, $j\left(g_{0}, p_{0}\right)=0.0158$. Row 2: intermediate state, shapes during line search \#4, cost $=0.00115$. Row 3: final state, shapes (after 5 line searches, $j\left(g_{\mathrm{fin}}, p_{\mathrm{fin}}\right)=1.00 \cdot 10^{-4}$, cost is reduced significantly, but precomputed optimal state of Fig. 1 is not quite recovered).

quite different: Convergence is reached only after 10 line searches and, thus, significantly slower than in Example 6.2(a). And even though the cost is reduced from 0.861 to 0.188 from initial to final state, the precomputed absolute min is not attained. While $\Omega_{g}$ does not change noticeably during the computation, the area of the difference between the final shape $\Omega_{p_{\text {fin }}}$ and the optimal shape is quite small (however, $\Omega_{p_{\text {fin }}}$ has more than the two connected components of the optimal shape). Comparing the isolevel pictures of the final states in Fig. 2 and Fig. 4, one observes 

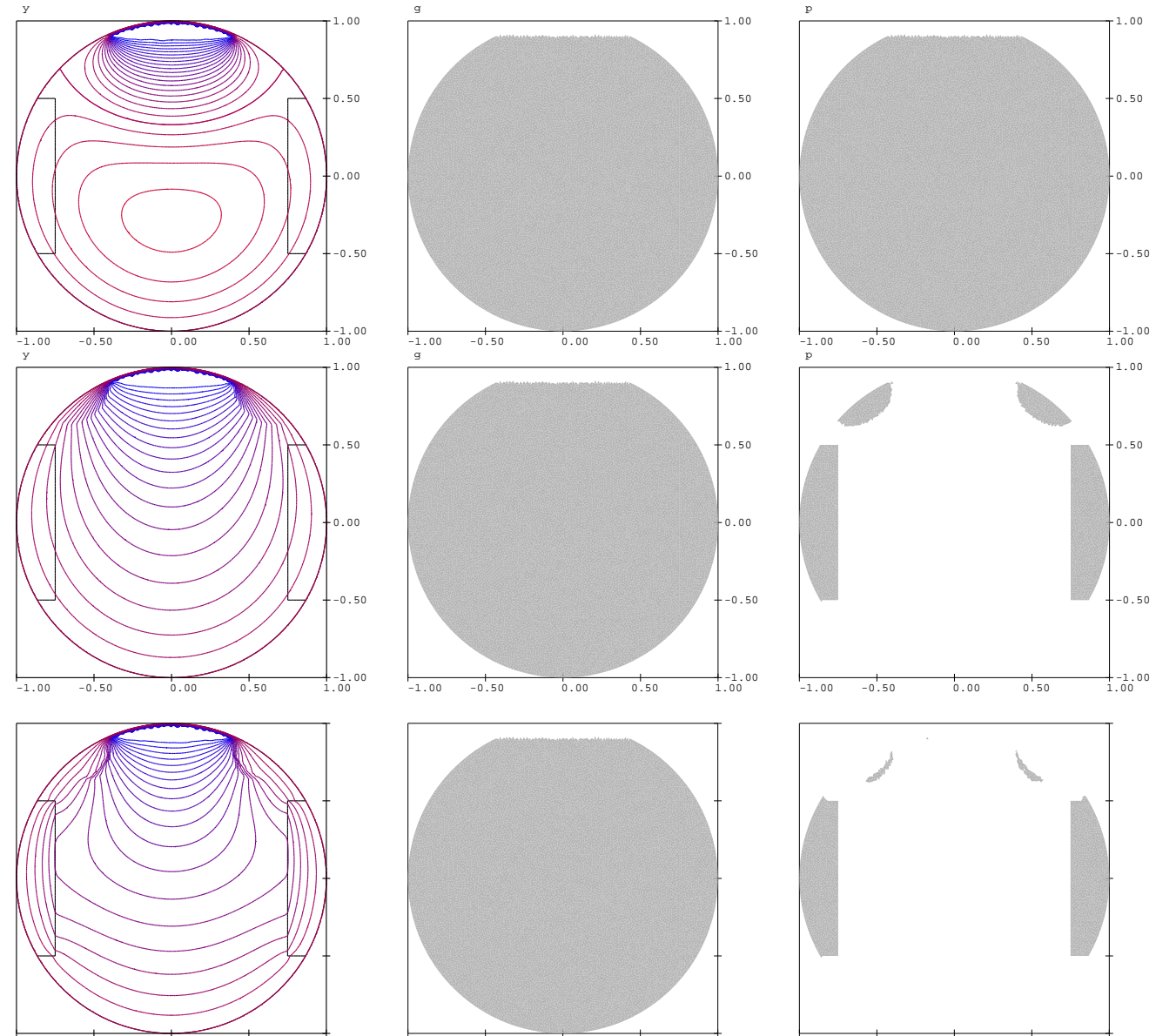

Figure 4: Shape optimization of Example 6.2(c). Left: State isolevels spaced at 0.1. Middle: Shapes $\Omega_{g}$ determined by $g \geq 0$ (in gray). Right: Shapes $\Omega_{p}$ determined by $p \geq 0$ (in gray). Row 1: initial state, shapes, $j\left(g_{0}, p_{0}\right)=0.861$. Row 2: intermediate state, shapes after line search \#1, $j\left(g_{1}, p_{1}\right)=0.415$. Row 3: final state, shapes (after 10 line searches, $j\left(g_{\text {fin }}, p_{\text {fin }}\right)=0.188$, precomputed optimal state of Fig. 1 has not been recovered).

that, in $E$, where the cost functional is active, the pictures almost agree, whereas, outside of $E$, they are completely different. Regarding symmetries, in contrast to previous examples, the $x_{1}$-axis symmetry is broken by the initial condition, and only the $x_{2}$-axis symmetry is retained.

(d) This example uses a different $y_{d}$ and a different right-hand side $f$. More precisely, the parameter settings are still as in (6.16), except for

$$
f\left(x_{1}, x_{2}\right):=-20\left(x_{1}^{2}+x_{2}^{2}\right)+20 \text {, }
$$


and the cost functional is as in (6.18), but now with

$$
y_{d}\left(x_{1}, x_{2}\right):=2
$$

Here, we are no longer in the situation of a known precomputed optimum.

As in (6.19), the initial shape functions are constantly 1 on $D$. Results are depicted in Fig. 5. In this case, most of the cost reduction and shape change occurs already during the first line search, with convergence being reached after 4 lines searches. While changes, including topological changes, occur in $\Omega_{p}$, the set $\Omega_{g}$ remains virtually constant.

Example 6.3. For the following numerical result, the fixed domain $D$ is still the unit disk as in (6.13). However, the fixed subdomain $E$ is now at the bottom of $D$,

$$
E:=\left\{\left(x_{1}, x_{2}\right) \in D: x_{2}<-0.7\right\} \subseteq D .
$$

The numerical computations employ a fixed triangular grid provided by Triangle [24, 25], consisting of 24623 triangles. The parameter settings are as in (6.16), except for

$$
f\left(x_{1}, x_{2}\right):=10\left(x_{1}^{2}+x_{2}^{2}\right)+5 \text {. }
$$

The cost functional is as in Example 6.2(d), i.e. (6.18) with $y_{d}\left(x_{1}, x_{2}\right):=2$. The initial shape functions are

$$
\begin{aligned}
& g_{0}\left(x_{1}, x_{2}\right):= \begin{cases}1 & \text { if } \sqrt{x_{1}^{2}+x_{2}^{2}} \geq 0.3, \\
-1 & \text { otherwise }\end{cases} \\
& p_{0}\left(x_{1}, x_{2}\right):= \begin{cases}1 & \text { if }\left(x_{1}, x_{2}\right) \in E, \\
-1 & \text { otherwise. }\end{cases}
\end{aligned}
$$



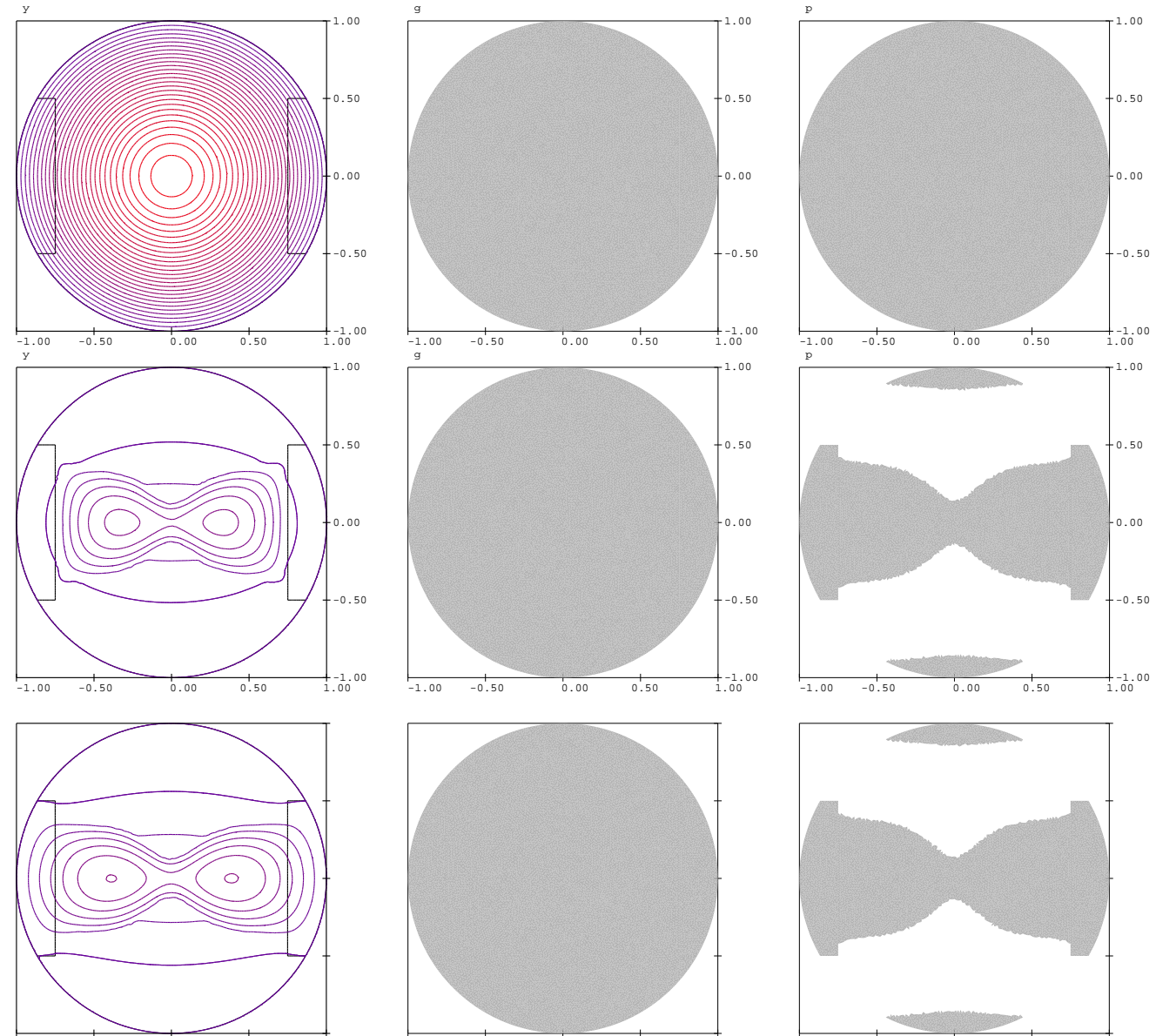

Figure 5: Shape optimization of Example 6.2(d). Left: State isolevels spaced at 0.1. Middle: Shapes $\Omega_{g}$ determined by $g \geq 0$ (in gray). Right: Shapes $\Omega_{p}$ determined by $p \geq 0$ (in gray). Row 1: initial state, shapes, $j\left(g_{0}, p_{0}\right)=2.97$. Row 2 : intermediate state, shapes after line search \#1, $j\left(g_{1}, p_{1}\right)=0.265$. Row 3 : final state, shapes (after 4 line searches, $\left.j\left(g_{\text {fin }}, p_{\text {fin }}\right)=0.262\right)$.

Results are depicted in Fig. 6. While, overall, one can observe similarities with the last case of Example 6.2, one now observes that the cost achieved during the first line search is actually lower than the final cost. Of course, this can occur only if $[g, p] \notin U_{\text {ad }}^{h}$ during the line search. Another difference to previous examples lies in the $x_{1}$-axis symmetry now being broken by the shape of $E$.

Acknowledgement. The second author acknowledges with thanks 

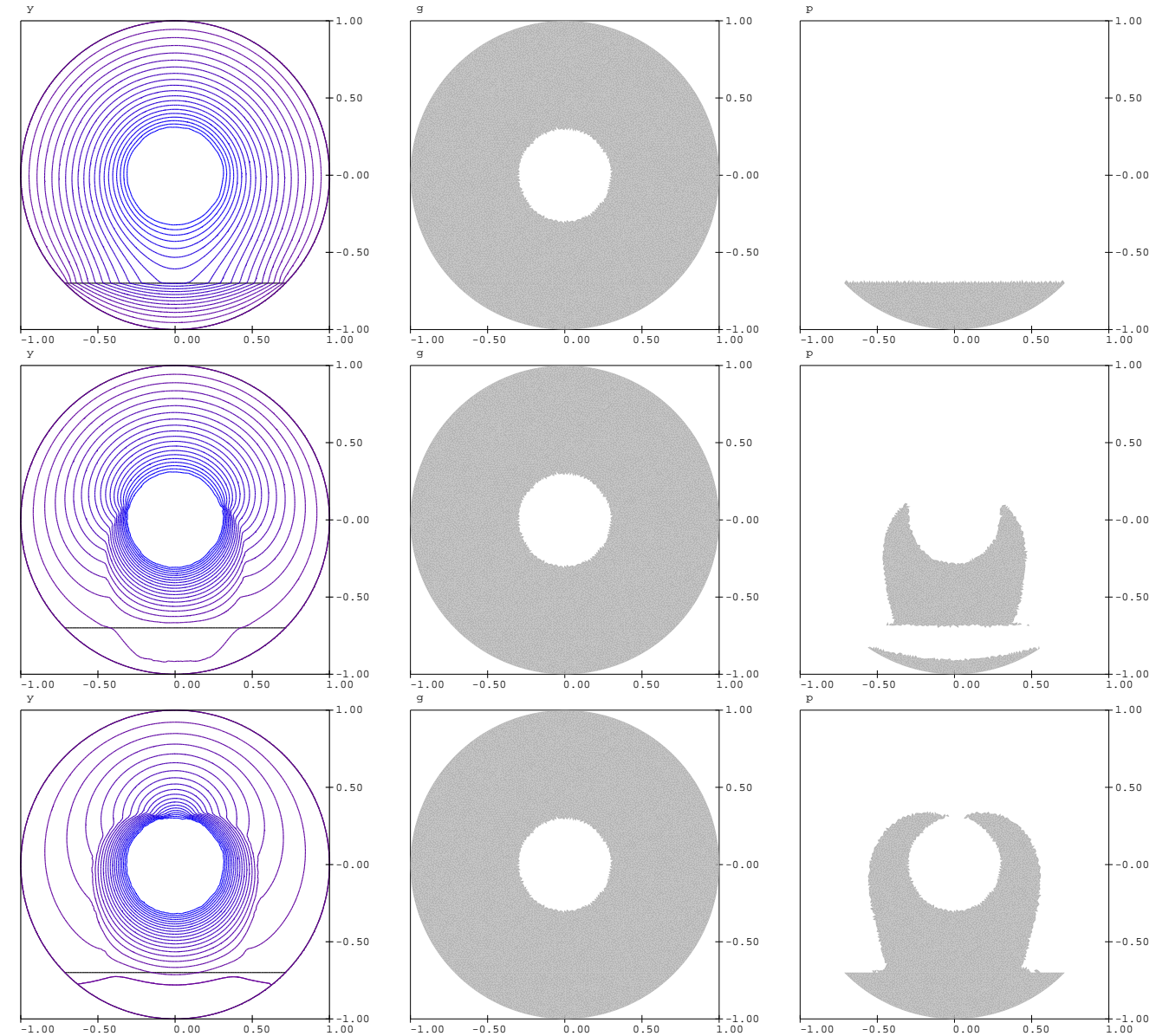

Figure 6: Shape optimization of Example 6.3. Left: State isolevels spaced at 0.1. Middle: Shapes $\Omega_{g}$ determined by $g \geq 0$ (in gray). Right: Shapes $\Omega_{p}$ determined by $p \geq 0$ (in gray). Row 1: initial state, shapes, $j\left(g_{0}, p_{0}\right)=2.75$. Row 2: intermediate state, shapes during line search $\# 1$, cost $=0.0842$. Row 3 : final state, shapes (after 3 line searches, $\left.j\left(g_{\text {fin }}, p_{\text {fin }}\right)=0.100\right)$.

very useful discussions with Prof. Enrique Zuazua on the analysis of such problems, and the financial support of Grant 145/2011, CNCS Romania.

\section{References}

[1] Allaire, G., Shape optimization by the Homogenization method, New York, Springer-Verlag (2001). 
[2] Bendsoe, M., Optimization of structural topology, shape, and material, Berlin, Springer-Verlag (1995).

[3] Brezis, H., Analyse Fonctionelle. Théorie et applications, Paris, Masson (1983).

[4] Casado-Diaz, J., Castro, C., Luna-Laynez, M., Zuazua, E., Numerical approximation of a one-dimensional elliptic optimal design problem, Multiscale Model. Simul. 9 (3) (2011), pp. 1181-1216.

[5] Chenais, D., Zuazua, E., Finite-element approximation of $2 D$ elliptic optimal design, J. Math. Pures Appl. 85 (9) (2006), pp. 225-249.

[6] Delfour, M.C., Zolesio, J.-P., Shapes and geometries. Analysis, differential calculus and optimization, SIAM, Philadelphia, PA (2001).

[7] Eymard, R., Gallouët, T., Herbin, R.: Finite Volume Methods, pp. 7131020 in Ciarlet, P., Lions, J. (eds.): Solution of Equations in $\mathbb{R}^{n}$ (Part 3); Techniques of Scientific Computing (Part 3), Handbook of Numerical Analysis, Vol. VII. North-Holland/ Elsevier, Amsterdam, The Netherlands (2000).

[8] Fuhrmann, J., Koprucki, T., Langmach, H.: pdelib: An open modular tool box for the numerical solution of partial differential equations. Design patterns. In: Hackbusch, W., Wittum, G. (eds.): Proceedings of the 14th GAMM Seminar on Concepts of Numerical Software, Kiel, January 2325, 1998. University of Kiel, Kiel, Germany (2001).

[9] Geiser, J., Klein, O., Philip, P.: Numerical simulation of temperature fields during the sublimation growth of SiC single crystals, using WIASHiTNIHS. J. Crystal Growth 303 (2007), pp. 352-356. 
[10] Grisvard, P., Elliptic problems in nonsmooth domains, Pitman, London (1985).

[11] Grund, F., Direct linear solvers for vector and parallel computers, pp. 114-127 in Vector and Parallel Processing VECPAR'98, Lecture Notes in Computer Science 1573 (1999).

[12] Klein, O., Lechner, C., Druet, P.-É, Philip, P., Sprekels, J., FrankRotsch, C., Kießling, F.-M., Miller, W., Rehse, U., Rudolph, P.: Numerical simulations of the influence of a traveling magnetic field, generated by an internal heater-magnet module, on liquid encapsulated Czochralski crystal growth. Magnetohydrodynamics 45 (2009), pp. 257-267. Special Issue: Selected papers of the International Scientific Colloquium Modelling for Electromagnetic Processing, Hannover, Germany, October 27$29,2008$.

[13] Mäkinen, R., Neittaanmäki, P., Tiba, D., On a fixed domain approach for a shape optimization problem, pp. 317-326 in Computational and applied mathematics II, Differential equations (Ames, W.F., van der Houwen, P.J., eds.), Amsterdam, North-Holland (1992).

[14] Meyers, N.G., An $L^{p}$ estimate for the gradient of solutions of second order elliptic divergence equations, Ann. Sc. Norm. Sup. Pisa 17 (3) (1963), pp. 189-206.

[15] Neittaanmäki, P., Pennanen, A., Tiba, D., Fixed domain approaches in shape optimization problems with Dirichlet boundary conditions, Inverse Problems 25 (2009), pp. 1-18.

[16] Neittaanmäki, P., Sprekels, J., Tiba, D., Optimization of elliptic systems. Theory and applications, New York, Springer-Verlag (2006). 
[17] Neittaanmäki, Tiba, D., Fixed domain approaches in shape optimization problems, accepted for publication in Inverse Problems. In press.

[18] Osher, S., Sethian, J.A., Fronts propagating with curvature-dependent speed: algorithms based on Hamilton-Jacobi formulations, J. Comput. Phys. 79 (1) (1988), pp. 12-49.

[19] Philip, P.: Analysis, optimal control, and simulation of conductiveradiative heat transfer. Mathematics and its Applications / Annals of AOSR 2 (2010), pp. 171-204.

[20] Philip, P., Tiba, D., Shape optimization via control of a shape function on a fixed domain: Theory and numerical results, pp. 285-299 in Numerical Methods for Differential Equations, Optimization, and Technological Problems, Computational Methods in Applied Sciences 27, Springer (2013) (in press).

[21] Pironneau, O., Optimal shape design for elliptic systems, Berlin, Springer (1984).

[22] Press, W., Teukolsky, S., Vetterling, W., Flannery, B.: Numerical Recipes. The Art of Scientific Computing, 3rd edn. Cambridge University Press, New York, USA (2007).

[23] Sethian, J.A., Level set methods, Cambridge MA, Cambridge Univ. Press (1996).

[24] Shewchuk, J.: Triangle: Engineering a 2D quality mesh generator and Delaunay triangulator, pp. 203-222 in Lin, M.C., Manocha, D. (eds.) Applied Computational Geometry: Towards Geometric Engineering, Lecture Notes in Computer Science 1148 (1996). 
[25] Shewchuk, J.: Delaunay refinement algorithms for triangular mesh generation. Computational Geometry: Theory and Applications 22 (2002), pp. 21-74.

[26] Sokolowsky, J., Zolesio, J.-P., Introduction to shape optimization. Shape sensitivity analysis, Berlin, Springer-Verlag (1992).

[27] Sverak, V., On optimal shape design, J. Math. Pures Appl. 72 (9) (1993), pp. 537-551.

[28] Tiba, D., Finite element approximation for shape optimization problems with Neumann and mixed boundary conditions, SIAM J. Control Optim. 49 (3) (2011), pp. 1064-1077.

[29] Woo, H., Kim, S., Seol, J.K., Lionheart, W., Woo, E.J., A direct tracking method for a grounded conductor inside a pipeline from capacitance measurements, Inverse Problems 22 (2006), pp. 481-494. 